\title{
Doblado de Papel y Software de Geometría Dinámica. Una Experiencia con Futuros Profesores de Matemática.
}

\author{
Martha Iglesias \\ mmiglesias@gmail.com \\ https://orcid.org/0000-0002-1800-0549 \\ Universidad Pedagógica Experimental Libertador. Instituto Pedagógico de Maracay (UPEL) \\ Maracay, Venezuela. \\ José Ortiz \\ ortizbuitrago@gmail.com \\ https://orcid.org/0000-0002-8396-7537 \\ Universidad de Carabobo. Campus La Morita (UC) \\ Maracay, Venezuela.
}

Recibido: 15/04/2020 Aceptado: 21/05/2020

\begin{abstract}
Resumen
El presente trabajo surge de un curso optativo de Resolución de Problemas Geométricos Asistido por Computadora (RPG-AC) que forma parte de la formación inicial de profesores de matemática en una universidad venezolana. Se estudian las actividades realizadas por 13 futuros profesores de matemática, en cinco grupos de trabajo, durante la implementación de un taller, el cual estuvo dirigido a la exploración de ángulos y triángulos con doblado de papel. Se utilizó el software de geometría dinámica CabriGéomètre II para mostrar la construcción con regla y compás de la herramienta triangular, y dejar ver su equivalencia con la construcción a partir del doblado de papel. En la validación de las construcciones realizadas en CabriGéomètre y en doblado de papel, se observaron esquemas de argumentación fácticos, empíricos y analíticos. Finalmente, el estudio realizado puso en evidencia que las actividades y experiencias formativas llevadas a cabo contribuyen a la formación didáctica y matemática de los futuros profesores.
\end{abstract}

Palabras clave: Formación inicial de profesores de matemática, educación secundaria, software de geometría dinámica, doblado de papel, esquemas de argumentación.

\section{Ambientes de geometria dinâmica e dobragem de papel. Uma experiência com futuros professores de matemática.}

\section{Resumo}

O presente trabalho nasce de um curso otátivo de Resoluçăo de Problemas Geométricos Assistido por Computador (RPG-AC) ó qual forma uma parte da formaçăo inicial de Professores de Matemática de uma Universidade venezuelana. Estudan-se as tarefas feitas por 13 (treze) futuros Professores de Matemática, em 5 (cinco) grupos de trabalho, durante a implementaçăo de um Talher, ó qual foi dirigido a exploraçăo de ângulos e triângulos com o dobrado de papel. Utilizou-se o software de Geometría dinámica: Cabri Géomètre II para ensinar a construçăo com régua e compás como ferramenta triângular, e deixar olhar a sua equivalènça com a construçăo desde o dobrado do papel. Na validaçăo das construçoěs feitas em Cabri y no dobrado do papel, olharon-se quadros de argumentaçăo fácticos, impíricos e 
analíticos. Por fim, o estudo realizado mostrou que as atividades e experiências de treinamento realizadas contribuem para a formação didática e matemática de futuros professores.

Palavras chave: formaçăo inicial de Professores de Matemática, Educaçăo secundária, software de Geometría dinámica, dobrado de papel, quadro de argumentaçăo.

\title{
Paper folding and dynamic geometry software. An experience with pre-service mathematics teachers
}

\begin{abstract}
This work arises from an optional course of Computer Aided Geometric Problem Solving (RPG-AC) that is part of the initial training of mathematics teachers in a Venezuelan university. The activities carried out by 13 future teachers of mathematics, in five working groups, are studied during the implementation of workshop, which was aimed at the exploration of angles and triangles with paper folding. The CabriGéomètre II dynamic geometry software was used to show the construction with the ruler and compass of the triangular tool, and to show its equivalence with the construction from the folded paper. In the validation of the constructions carried out in CabriGéomètre and in paper folding, factual, empirical and analytical argumentation schemes were observed. Finally, this study showed that the training activities and experiences carried out contribute to the didactic and mathematical training to pre-service teachers.
\end{abstract}

Keywords: Pre-service mathematics teachers, secondary school, dynamical geometry software, folded paper, argumentation schemes.

\section{Introducción}

En el contexto de la formación inicial de profesores de Matemática en una universidad pública venezolana, se ha venido administrando un curso optativo de formación especializada de Resolución de Problemas Geométricos Asistido por Computadora (RPG-AC), el cual ha servido de escenario formativo e investigativo en el área de Geometría y su Didáctica. Dicho curso se centra básicamente en dos tipos de actividades: Talleres de resolución de problemas geométricos haciendo uso de un software de Geometría Dinámica como lo es el CabriGéomètre II y el diseño de unidades didácticas con contenidos geométricos contemplados en los programas de estudios de Matemática para la educación media en Venezuela; pretendiendo con ello propiciar la puesta en práctica por parte de los docentes en formación de competencias didáctico-matemáticas asociadas al proceso de enseñanza y aprendizaje de la Geometría. En Iglesias y Ortiz (2019), se presenta una caracterización, desde una perspectiva didáctica, de las experiencias de aprendizaje que han conformado el mencionado curso; específicamente el caso de experiencias vinculadas a la demostración en Geometría, a partir de la resolución de problemas haciendo uso del Cabri II. 
Cabe señalar que las actividades planteadas en los talleres de resolución de problemas geométricos han sido organizadas atendiendo a las fases de aprendizaje propuestas en el modelo de razonamiento geométrico (Van Hiele, 1957, 1959; Gutiérrez y Jaime, 2012): Información, orientación guiada o dirigida, explicitación, orientación libre e integración, con énfasis en las construcciones geométricas con regla y compás y siguiendo el esquema didáctico-matemático: Construir $\rightarrow$ Explorar $\rightarrow$ Conjeturar $\rightarrow$ Validar, el cual ha sido considerado por otros educadores matemáticos como Alsina Catalá, Fortuny Aymemí y Pérez Gómez (1997), Perry Carrasco, Camargo Uribe, Samper de Caicedo y Rojas Morales (2006) y Flores (2007) tanto para planificar tareas con contenido geométrico mediadas por tecnología como para analizar las producciones de sus estudiantes.

Este artículo tiene como propósito analizar y dar a conocer las actividades y experiencias formativas llevadas a cabo por un grupo de trece (13) estudiantes, cuatro (4) hombres y nueve (9) mujeres, cursantes del quinto semestre de la especialidad de Matemática en la Universidad Pedagógica Experimental Libertador, Instituto Pedagógico de Maracay (UPEL, IP. de Maracay) durante el desarrollo del taller $\mathrm{n}^{\circ} 2$; cabe decir que, para la implementación de las actividades planteadas en la correspondiente hoja de trabajo, los participantes se organizaron en seis (6) pequeños grupos de trabajo.

Las actividades planteadas en el taller $\mathrm{n}^{\circ} 2$ se sustentaron en lo propuesto por Arrieche e Iglesias (2010), quienes presentaron una herramienta didáctica construida mediante el doblado de papel, la cual puede ser utilizada para medir ciertos ángulos, cuando no se cuenta con un transportador; asimismo, se mostró que puede ser aprovechada para reforzar el estudio de las nociones de ángulos y triángulos y propiciar el desarrollo de algunas habilidades geométricas en los estudiantes. Además, emplearon el Cabri II para mostrar la construcción con regla y compás de esta herramienta triangular, basándose en la trisección de un ángulo recto; procurando describir el procedimiento empleado y dejando ver su equivalencia con la construcción con doblado de papel.

Por ello, en el taller $n^{\circ} 2$ del curso de Resolución de Problemas Geométricos Asistido por Computadora (RPG-AC), las actividades dirigidas estuvieron centradas en la construcción de tal herramienta triangular tanto con doblado de papel como con regla y compás en un ambiente de Geometría Dinámica (AGD), a partir de la lectura y análisis del mencionado artículo. En cuanto a las actividades libres, los participantes seleccionaron una figura 
geométrica y realizaron su construcción de ambas maneras, tratando de establecer la equivalencia entre ambas construcciones.

Para llevar a cabo el análisis del razonamiento seguido por los participantes del curso de RPG-AC, cuando efectuaron las construcciones de figuras geométricas con doblado de papel y un software de geometría dinámica, se han teniendo en cuentas las acciones realizadas por ellos: construcción, exploración, formulación de conjeturas y validación, enfatizando en la justificación como práctica argumentativa, la cual puede ser vista como una actividad intelectual en la cual se ponen en juego lo que Flores (2007) denomina esquemas de argumentación. Para Flores, la justificación de un resultado es el proceso de validación de éste y establece que la demostración o prueba - no hace distinciones entre estos términos - puede ser entendida como resultado de una práctica argumentativa que se apoya en esquemas analíticos cuyos razonamientos son válidos; por lo cual, se ha dedicado a estudiar los esquemas de argumentación en profesores de Matemáticas del bachillerato mexicano. Según Flores, se entiende por esquema de argumentación a la manera en que una persona utiliza sus razonamientos durante una práctica argumentativa dirigida a justificar o explicar un resultado o para validar una conjetura surgida durante el proceso de resolución de un problema. A continuación, se describen los tipos de esquemas de argumentación identificados por Flores (2007):

-Autoritarios: Sus argumentaciones se apoyan en las afirmaciones hechas por alguna autoridad; en este caso, puede ser un compañero, un libro de texto o el facilitador del curso.

-Simbólicos: En los que el profesor en formación utiliza un lenguaje matemático y símbolos de manera superflua y poco consistente, sin llegar realmente a las conclusiones a las que quiere llegar. En este tipo de esquemas pueden mencionar conceptos poco claros o inventados.

-Fácticos: En los que el profesor en formación hace un recuento de lo que hizo o repite hechos evidentes de una situación a manera de explicación o justificación de algún resultado. A menudo, se limita a exponer una serie de pasos como si fueran un algoritmo.

-Empíricos: En los que el profesor en formación se apoya en hechos físicos o en un dibujo. En este caso, el dibujo constituye un argumento por sí mismo y no un apoyo para visualizar un argumento. 
- Analíticos: En los que el profesor en formación sigue una cadena deductiva, sin que por ello llegue forzosamente a una conclusión válida.

Seguidamente, se analizará la fundamentación geométrica de las construcciones con doblado de papel, a partir de la revisión de los axiomas de la papiroflexia propuestos por Germán Luis Beitia, Humiaki Huzita y Roger Alperin y, posteriormente, teniendo en cuenta las producciones de los participantes en el curso de RPG-AC, se ilustrará la equivalencia entre dos maneras distintas de construir una figura geométrica y los esquemas de argumentación puestos en juego por un grupo de profesores en formación.

\section{Axiomas que se siguen en el doblado de papel}

Es necesario destacar que, en este caso, el doblado de papel fue utilizado con fines educativos (construcción de figuras planas para el estudio de sus propiedades geométricas) y no artísticos (construcción de figuras o cuerpos geométricos con fines ornamentales); el doblado se realizó a partir de un trozo de papel en forma rectangular, cuadrada o triangular y no a partir de tiras.

Para entender la noción de equivalencia entre una construcción con doblado de papel y una construcción con regla y compás de un mismo objeto geométrico, es preciso tener en cuenta los axiomas con los que se trabaja en el doblado de papel o la papiroflexia. En la Tabla 1, teniendo en cuenta la investigación realizada por Sánchez (2008), se mencionan los axiomas propuestos por Germán Luis Beitia, HumiakiHuzita y Roger Alperin.

Nótese, en el Cuadro 1, que estos tres conjuntos de axiomas del doblado de papel guardan relación con propiedades (axiomas o teoremas) de la Geometría Euclidiana, pero pudiera decirse que se basan en tres ideas matemáticas distintas, pero relacionadas entre sí: (a) superposición de figuras geométricas (Cuadro 2); (b) correspondencia entre figuras geométricas (Cuadro 3), y (c) puntos y rectas construibles (Cuadro 4). 
Cuadro 1: Axiomas en los cuales se soporta el doblado de papel o papiroflexia

\begin{tabular}{|c|c|c|}
\hline $\begin{array}{ll}\text { I. } & \text { Germán Luis } \\
& \text { Beitia }\end{array}$ & Humiaki Huzita & Roger Alperin \\
\hline $\begin{array}{l}\text { Puede considerarse que una } \\
\text { hoja es una superficie plana. }\end{array}$ & $\begin{array}{l}\text { Dados dos puntos distintos } \mathrm{P}_{1} \mathrm{y} \\
\mathrm{P}_{2} \text {, se puede realizar un pliegue } \\
\text { que los conecte. }\end{array}$ & $\begin{array}{l}\text { La línea que conecta dos puntos } \\
\text { construibles es una línea } \\
\text { construible. }\end{array}$ \\
\hline $\begin{array}{l}\text { Un pliegue realizado en una } \\
\text { hoja de papel que pase por dos } \\
\text { puntos y que se ha hecho sobre } \\
\text { una superficie plana como } \\
\text { soporte es una línea recta. }\end{array}$ & $\begin{array}{l}\text { Dados dos puntos distintos } \mathrm{P}_{1} \mathrm{y} \\
\mathrm{P}_{2} \text {, podemos plegar } \mathrm{P}_{1} \text { sobre } \mathrm{P}_{2} \text {. }\end{array}$ & $\begin{array}{l}\text { El punto de coincidencia de dos } \\
\text { líneas construibles es un punto } \\
\text { construible. }\end{array}$ \\
\hline $\begin{array}{l}\text { El papel puede ser plegado de } \\
\text { tal manera que pase por dos o } \\
\text { más puntos colineales. }\end{array}$ & $\begin{array}{l}\text { Dadas dos rectas distintas } \mathrm{L}_{1} \mathrm{y} \\
\mathrm{L}_{2} \text {, podemos plegar } \mathrm{L}_{1} \text { sobre } \mathrm{L}_{2} \text {. }\end{array}$ & $\begin{array}{l}\text { La mediatriz de un segmento } \\
\text { que conecta dos puntos } \\
\text { construibles es una línea } \\
\text { construible. }\end{array}$ \\
\hline $\begin{array}{l}\text { Puede superponerse dos puntos } \\
\text { distintos en una misma hoja de } \\
\text { papel. }\end{array}$ & $\begin{array}{l}\text { Dado un punto } \mathrm{P} \text { y una recta } \mathrm{L} \text {, } \\
\text { podemos hacer un pliegue } \\
\text { perpendicular a } \mathrm{L} \text { que pase por } \\
\mathrm{P} \text {. }\end{array}$ & $\begin{array}{l}\text { La línea que biseca cualquier } \\
\text { ángulo dado puede ser } \\
\text { construida. }\end{array}$ \\
\hline $\begin{array}{l}\text { Puede doblarse el papel de } \\
\text { modo que un punto puede } \\
\text { superponerse a otro pliegue. }\end{array}$ & $\begin{array}{l}\text { Dados dos puntos distintos } \mathrm{P}_{1} \mathrm{y} \\
\mathrm{P}_{2} \mathrm{y} \text { una recta } \mathrm{L} \text {, podemos hacer } \\
\text { un pliegue que haga } \\
\text { corresponder a } \mathrm{P}_{1} \text { con un punto } \\
\text { de } \mathrm{L} \text { y que pase por } \mathrm{P}_{2} \text {. }\end{array}$ & $\begin{array}{l}\text { Dada una línea construida L y } \\
\text { los puntos construidos } \mathrm{P} \text { y Q, } \\
\text { entonces siempre es posible } \\
\text { construir la línea que pasa por } \\
\text { Q y que refleja a P en L. }\end{array}$ \\
\hline $\begin{array}{l}\text { Puede plegarse el papel de } \\
\text { modo que dos pliegues de una } \\
\text { misma hoja pueden } \\
\text { superponerse. }\end{array}$ & $\begin{array}{l}\text { Dados dos puntos distintos } \mathrm{P}_{1} \mathrm{y} \\
\mathrm{P}_{2} \mathrm{y} \text { dos rectas distintas } \mathrm{L}_{1} \mathrm{y} \mathrm{L}_{2} \text {, } \\
\text { podemos hacer un pliegue que } \\
\text { haga corresponder a } \mathrm{P}_{1} \text { con un } \\
\text { punto de } \mathrm{L}_{1} \text { y } \mathrm{P}_{2} \text { con un punto } \\
\text { de } \mathrm{L}_{2} \text {. }\end{array}$ & $\begin{array}{l}\text { Dadas las líneas construidas L y } \\
\text { M y los puntos construidos P y } \\
\text { Q, entonces siempre es posible } \\
\text { construir una línea que } \\
\text { simultáneamente refleja a P en } \\
\text { L y a Q en M. }\end{array}$ \\
\hline \multicolumn{3}{|l|}{$\begin{array}{l}\text { Dos ángulos son congruentes, si } \\
\text { al superponerse coinciden. }\end{array}$} \\
\hline $\begin{array}{l}\text { Dos segmentos son congruentes } \\
\text { si al superponerse coinciden. }\end{array}$ & & \\
\hline
\end{tabular}

En este primer conjunto de axiomas del doblado de papel, mencionados en el Cuadro 2, la idea clave es la de superposición de figuras geométricas, entendida como la técnica empleada desde la antigua Grecia para comparar dos figuras geométricas en cuanto a forma y tamaño y así decidir si eran congruentes o no, ya que, mediante la superposición de dos figuras que coinciden se prueba que las mismas son congruentes. 
Cuadro 2. Relación de los axiomas propuestos por Beitia con propiedades de la Geometría Euclidiana

\begin{tabular}{|c|c|}
\hline $\begin{array}{c}\text { Axiomas propuestos por German } \\
\text { Luis Beitia }\end{array}$ & Definiciones, axiomas y teoremas de la Geometría Euclidiana \\
\hline $\begin{array}{l}\text { Puede considerarse que una } \\
\text { hoja es una superficie plana. }\end{array}$ & \multirow{3}{*}{$\begin{array}{l}\text { En la Geometría Euclidiana se consideran como términos primitivos o } \\
\text { términos no definidos a los conceptos de punto, recta y plano. De modo que } \\
\text { el espacio es el conjunto de todos los puntos y las rectas y los planos son } \\
\text { subconjuntos notables del espacio. Suele hablarse del espacio } \\
\text { tridimensional. Asimismo, el Axioma o Postulado de la Recta establece que } \\
\text { dos puntos distintos cualesquiera determinan una recta y solamente una; es } \\
\text { decir, dados dos puntos distintos A y B, existe exactamente una recta que } \\
\text { pasa por tales puntos y, por ello, se denota por } \overleftrightarrow{A B} \text {. De modo que, si un } \\
\text { punto C pertenece a la recta AB, puede escribirse que } \overleftrightarrow{A B}=\overleftrightarrow{B C}=\overleftrightarrow{A C} \\
\text { y, además, decirse que los puntos A, B y Cestán alineados o que son puntos } \\
\text { colineales. Además, el Axioma o el Postulado del Plano establece que tres } \\
\text { puntos distintos cualesquiera no alineados determinan un único plano; en } \\
\text { consecuencia, dada una recta L y un punto P no perteneciente a L, tales } \\
\text { objetos determinan un único plano. También se conoce que si dos puntos } \\
\text { distintos cualesquiera pertenecen a un plano, entonces la recta que pasa } \\
\text { por ellos está contenida en el plano. Esto nos garantiza que los planos son } \\
\text { llanos. Por ende, si una recta L interseca a un plano E, que no la contiene, } \\
\text { su intersección es un punto solamente. Por otra parte, si dos rectas distintas } \\
\text { se intersecan, su intersección es un punto solamente y, además, tales rectas } \\
\text { determinan un único plano. }\end{array}$} \\
\hline $\begin{array}{l}\text { Un pliegue realizado en una } \\
\text { hoja de papel que pase por dos } \\
\text { puntos y que se ha hecho sobre } \\
\text { una superficie plana como soporte } \\
\text { es una línea recta. }\end{array}$ & \\
\hline $\begin{array}{l}\text { El papel puede ser plegado de } \\
\text { tal manera que pase por dos o más } \\
\text { puntos colineales. }\end{array}$ & \\
\hline $\begin{array}{l}\text { Puede superponerse dos puntos } \\
\text { distintos en una misma hoja de } \\
\text { papel. }\end{array}$ & \multirow[t]{3}{*}{$\begin{array}{l}\text { La superposición de dos figuras es la técnica desarrollada por los antiguos } \\
\text { geómetras griegos para comparar figuras geométricas en cuanto a forma y } \\
\text { tamaño. }\end{array}$} \\
\hline $\begin{array}{l}\text { Puede doblarse el papel de } \\
\text { modo que un punto puede } \\
\text { superponerse a otro pliegue. }\end{array}$ & \\
\hline $\begin{array}{l}\text { Puede plegarse el papel de } \\
\text { modo que dos pliegues de una } \\
\text { misma hoja pueden superponerse. }\end{array}$ & \\
\hline $\begin{array}{l}\text { Dos ángulos son congruentes, } \\
\text { si al superponerse coinciden. }\end{array}$ & \multirow{2}{*}{$\begin{array}{l}\text { Dos figuras geométricas son congruentes, si tienen la misma forma y el } \\
\text { mismo tamaño; por ello, al superponer una sobre la otra, coinciden; es } \\
\text { decir, no sobra, ni falta algo. En este caso, dos ángulos son congruentes, si } \\
\text { tienen la misma medida y dos segmentos son congruentes, si tienen la } \\
\text { misma longitud. }\end{array}$} \\
\hline $\begin{array}{l}\text { Dos segmentos son } \\
\text { congruentes si al superponerse } \\
\text { coinciden. }\end{array}$ & \\
\hline
\end{tabular}


Cuadro 3. Relación de los axiomas propuestos por Huzita con propiedades de la Geometría Euclidiana

\begin{tabular}{|c|c|}
\hline $\begin{array}{l}\text { Axiomas propuestos } \\
\text { por Humiaki Huzita }\end{array}$ & Definiciones, axiomas y teoremas de la Geometría Euclidiana \\
\hline $\begin{array}{l}\text { Dados dos puntos } \\
\text { distintos } \mathrm{P}_{1} \text { y } \mathrm{P}_{2} \text {, se } \\
\text { puede realizar un } \\
\text { pliegue que los conecte. }\end{array}$ & $\begin{array}{l}\text { Axioma o Postulado de la Recta: Por dos puntos distintos cualesquiera pasa una } \\
\text { única recta o dos puntos distintos cualesquiera determinan una única recta. }\end{array}$ \\
\hline $\begin{array}{l}\text { Dados dos puntos } \\
\text { distintos } \mathrm{P}_{1} \text { y } \mathrm{P}_{2} \\
\text { podemos plegar } \mathrm{P}_{1} \\
\text { sobre } \mathrm{P}_{2} \text {. }\end{array}$ & $\begin{array}{l}\text { Dado que dos puntos distintos A y } \mathrm{B} \text { determinan una única recta } \overleftrightarrow{A B} \text {, así como un } \\
\text { segmento } \overline{A B} \text { contenido en dicha recta. Además, el punto medio } \mathrm{M} \text { de un segmento } \\
\overline{A B} \text { existe y es único y la mediatriz del segmento } \overline{A B} \text { es la recta perpendicular al } \\
\text { segmento en su punto medio. Nótese que al hacer coincidir } \mathrm{P}_{1} \text { con } \mathrm{P}_{2} \text {, con doblado } \\
\text { de papel, el pliegue representa la mediatriz del segmento } \bar{P}_{1} P_{2}\end{array}$ \\
\hline $\begin{array}{l}\text { Dadas dos rectas } \\
\text { distintas } \mathrm{L}_{1} \text { y } \mathrm{L}_{2}, \\
\text { podemos plegar } \mathrm{L}_{1} \\
\text { sobre } \mathrm{L}_{2} \text {. }\end{array}$ & $\begin{array}{l}\text { Dado que } \mathrm{L}_{1} \text { y } \mathrm{L}_{2} \text { son coplanarias, caben dos opciones: (a) que se intersequen } \\
\text { (rectas secantes) y su intersección es un punto solamente; determinándose así } \\
\text { cuatro ángulos (dos pares de ángulos opuestos por el vértice), de modo que al hacer } \\
\text { coincidir una recta con la otra, se bisecan dos de estos ángulos. (b) que no se } \\
\text { intersequen (rectas paralelas), al hacer coincidir una recta con la otra, el pliegue } \\
\text { representa una recta } \mathrm{L}_{3} \text { paralela a las rectas } \mathrm{L}_{1} \text { y } \mathrm{L}_{2} \text {. }\end{array}$ \\
\hline $\begin{array}{l}\text { Dado un punto } P \text { y una } \\
\text { recta } L \text {, podemos hacer } \\
\text { un pliegue } \\
\text { perpendicular a } L \text { que } \\
\text { pase por } P \text {. }\end{array}$ & $\begin{array}{l}\text { Dados un punto } \mathrm{P} \text { y una recta } \mathrm{L} \text {, por } \mathrm{P} \text { pasa una y solo una recta } \mathrm{L}_{1} \text { perpendicular a } \\
\mathrm{L} \text {. Nótese que } \mathrm{P} \text { puede pertenecer o no a la recta } \mathrm{L} \text {. }\end{array}$ \\
\hline $\begin{array}{l}\text { Dados dos puntos } \\
\text { distintos } P_{1} \text { y } P_{2} \text { y una } \\
\text { recta } L \text {, podemos hacer } \\
\text { un pliegue que haga } \\
\text { corresponder a } P_{1} \text { con } \\
\text { un punto de } L \text { y que } \\
\text { pase por } P_{2} \text {. }\end{array}$ & 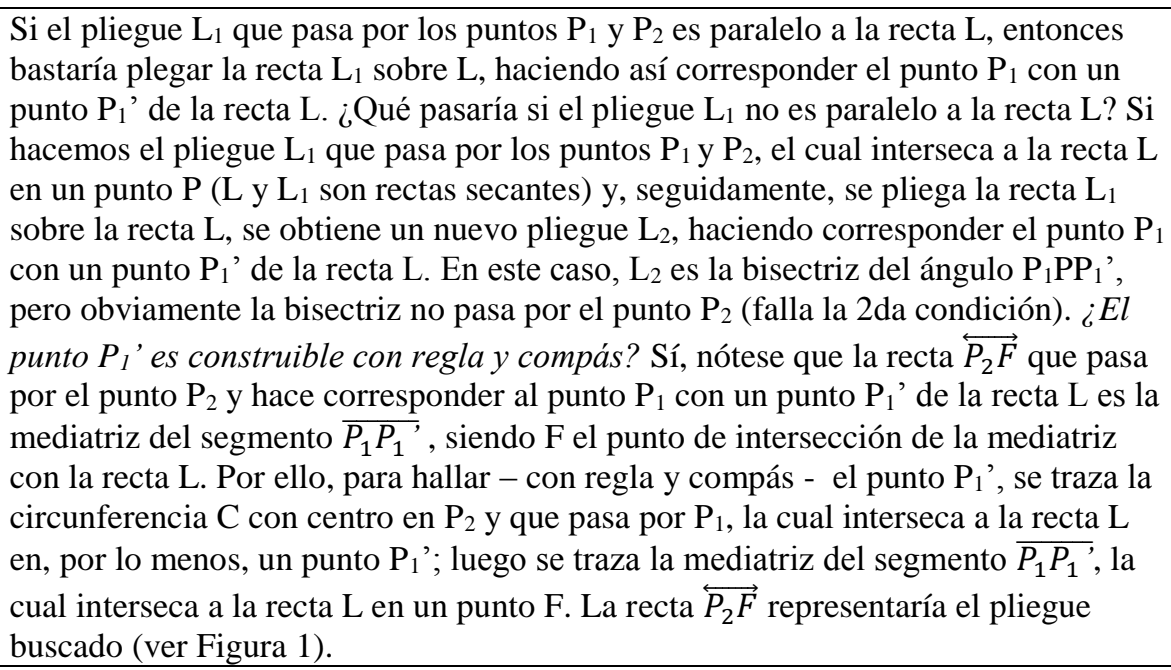 \\
\hline $\begin{array}{l}\text { Dados dos puntos } \\
\text { distintos } \mathrm{P}_{1} \text { y } \mathrm{P}_{2} \text { y dos } \\
\text { rectas distintas } \mathrm{L}_{1} \text { y } \mathrm{L}_{2} \text {, } \\
\text { podemos hacer un } \\
\text { pliegue que haga } \\
\text { corresponder a } \mathrm{P}_{1} \text { con } \\
\text { un punto de } \mathrm{L}_{1} \text { y } \mathrm{P}_{2} \text { con } \\
\text { un punto de } \mathrm{L}_{2} \text {. }\end{array}$ & $\begin{array}{l}\text { Avilés Fajardo (2016) señala que "la aplicación de este postulado se relaciona con } \\
\text { la solución de un problema del cálculo, consistente en encontrar una recta tangente } \\
\text { que sea tangente a dos parábolas" (p. 22); es decir, un problema que consiste en } \\
\text { hallar la recta que sea tangente tanto a la parábola cuya directriz es } L_{1} \text { y cuyo foco } \\
\text { es } P_{1} \text { como a la parábola cuya directriz es } L_{2} \text { y cuyo foco es } P_{2} \text {. }\end{array}$ \\
\hline
\end{tabular}

En este segundo conjunto de axiomas, señalados en el Cuadro 3, se asume que el plano (representado por la hoja de papel) es un conjunto de infinitos puntos y que es factible, mediante operaciones propias del doblado de papel, establecer una correspondencia uno a uno 
entre los puntos pertenecientes a dicho plano. Cabe señalar que Chocarro Pernaut (2016) realizó un estudio matemático de los llamados Axiomas de Huzita, probando que se trata de un conjunto completo de axiomas, ya que, "cualquier pliegue que podamos realizar será una construcción incluida en los axiomas" (p.3). La discusión sobre este estudio trasciende al propósito de este artículo, pero se sugiere su revisión para profundizar en la fundamentación matemática del doblado de papel o papiroflexia. En este orden de ideas, Royo Prieto (2002) señala que "los puntos construibles con regla y compás son exactamente los mismos que los construibles con los cinco primeros axiomas"(de Huzita) (p. 188). Además, Avilés Fajardo (2016) indica que, a los seis axiomas iniciales propuestos por Huzita, se ha incorporado un séptimo axioma (considerado por Chocarro Pernaut en su estudio): Dados un punto $\mathrm{P}_{1} \mathrm{y}$ dos rectas distintas $L_{1} \mathrm{y} \mathrm{L}_{2}$, se puede hacer un pliegue o doblez perpendicular a $\mathrm{L}_{2}$ que haga corresponder el punto $\mathrm{P}_{1}$ con un punto de $\mathrm{L}_{1}$; problema que consiste en "hallar un doblez que sea tangente a la parábola cuya directriz es $\mathrm{L}_{1}$ y cuyo foco es $\mathrm{P}_{1}$, e igualmente, sea perpendicular a la recta que determina el doblez $\mathrm{L}_{2}$ ” (p. 22).

Figura 1. Interpretación geométrica del Axioma 5 de los propuestos por Huzita

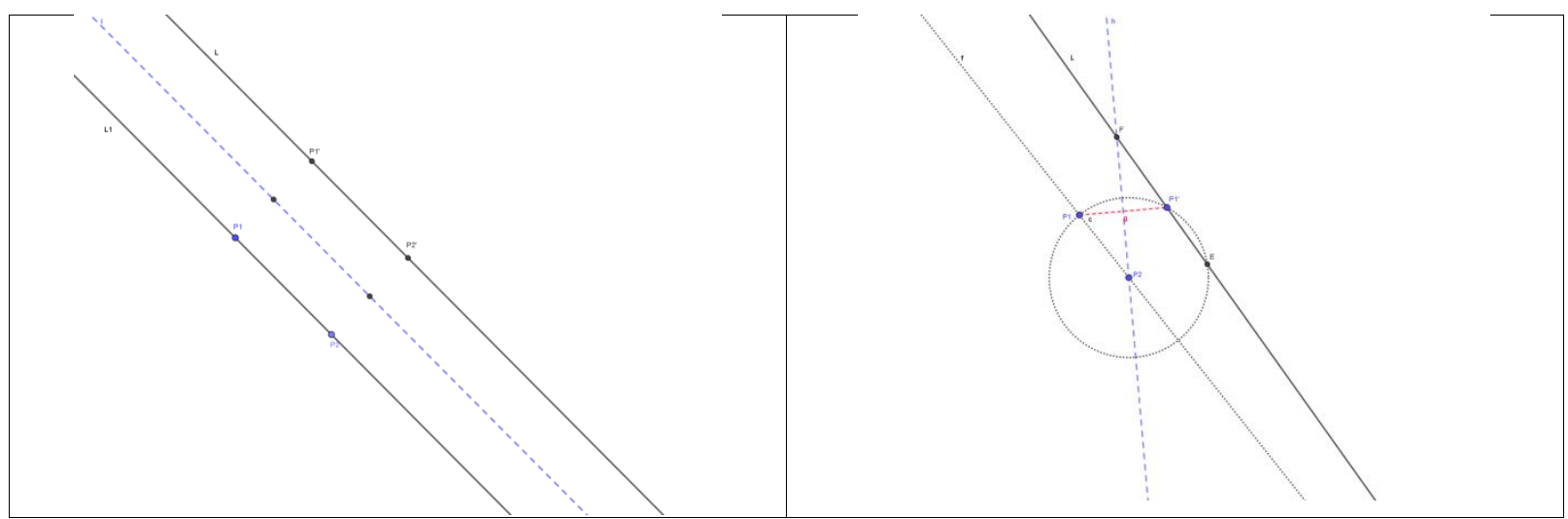

Fuente: Elaboración Propia

En el tercer conjunto de axiomas (ver Cuadro 4), es clave la noción de puntos y rectas construibles, la cual es también propia de las construcciones con regla y compás, donde un punto es construible por la intersección de dos rectas distintas, o por la intersección de una recta con un arco de circunferencia, o por la intersección de dos arcos de circunferencia, o por un número finito de estas operaciones. 
Cuadro 4. Relación de los axiomas propuestos por Alperin con propiedades de la Geometría Euclidiana

\begin{tabular}{|c|c|}
\hline $\begin{array}{l}\text { Axiomas propuestos por Roger } \\
\text { Alperin }\end{array}$ & Definiciones, axiomas y teoremas de la Geometría Euclidiana \\
\hline $\begin{array}{l}\text { La línea que conecta dos puntos } \\
\text { construibles es una línea } \\
\text { construible. }\end{array}$ & $\begin{array}{l}\text { Axioma o Postulado de la Recta: Por dos puntos distintos } \\
\text { cualesquiera pasa una única recta o dos puntos distintos } \\
\text { cualesquiera determinan una única recta. }\end{array}$ \\
\hline $\begin{array}{l}\text { El punto de coincidencia de dos } \\
\text { líneas construibles es un punto } \\
\text { construible. }\end{array}$ & $\begin{array}{l}\text { Si dos rectas distintas se intersecan, su intersección es un punto } \\
\text { solamente y, además, determinan un único plano. }\end{array}$ \\
\hline $\begin{array}{l}\text { La mediatriz de un segmento } \\
\text { que conecta dos puntos } \\
\text { construibles es una línea } \\
\text { construible. }\end{array}$ & $\begin{array}{l}\text { La mediatriz de un segmento es la recta perpendicular al segmento } \\
\text { en su punto medio. }\end{array}$ \\
\hline $\begin{array}{l}\text { La línea que biseca cualquier } \\
\text { ángulo dado puede ser } \\
\text { construida. }\end{array}$ & $\begin{array}{l}\text { Dados un ángulo }<\mathrm{AOB} \text { y } \mathrm{C} \text { un punto perteneciente al interior del } \\
<\mathrm{AOB} \text {. Se dice que la semirrecta } \overrightarrow{O C} \text { es la bisectriz del ángulo } \\
\text { dado, si } \mathrm{m}(<\mathrm{AOC})=\mathrm{m}(<\mathrm{COB}) \text {. También se dice que la } \\
\text { semirrecta } \overrightarrow{O C} \text { biseca al }<\mathrm{AOB} \text {. }\end{array}$ \\
\hline $\begin{array}{l}\text { Dada una línea construida L y } \\
\text { los puntos construidos P y Q, } \\
\text { entonces siempre es posible } \\
\text { construir la línea que pasa por } \\
\text { Q y que refleja a P en L. }\end{array}$ & \multirow{2}{*}{$\begin{array}{l}\text { Estos axiomas se corresponden con los axiomas } 5 \text { y } 6 \text { propuestos } \\
\text { por Huzita; según Royo Prieto (2002), en un artículo publicado por } \\
\text { Roger Alperin en el año 2000, este autor realiza una discusión del } \\
\text { alcance de los axiomas presentados y, además, caracteriza a "los } \\
\text { puntos del origami como aquellos puntos del plano complejo C } \\
\text { construibles tras la aplicación finita de los axiomas" (aquí } \\
\text { señalados) (p. 188). }\end{array}$} \\
\hline $\begin{array}{l}\text { Dadas las líneas construidas L y } \\
\text { M y los puntos construidos P y } \\
\text { Q, entonces siempre es posible } \\
\text { construir una línea que } \\
\text { simultáneamente refleja a P en } \\
\text { L y a Q en M. }\end{array}$ & \\
\hline
\end{tabular}

Fuente: Elaboración Propia

Cabe decir que la clave de lo hasta aquí señalado, desde un punto de vista tanto matemático como didáctico, es la interpretación geométrica de lo que se hace cuando realizamos una construcción tanto con doblado de papel como con regla y compás y, más, cuando se trata de establecer equivalencia entre el procedimiento empleado en cada caso.

En el curso de Resolución de Problemas Geométricos Asistido por Computadora (RPGAC), para la construcción de figuras geométricas con doblado de papel se tomaron en consideración las siguientes "reglas":

-Una hoja de papel - independientemente de su forma - es asumida como una superficie plana que posee infinitos puntos.

-Un pliegue realizado en una hoja de papel que pase por dos puntos es asumido como una línea recta.

-El papel puede ser plegado de tal manera que el pliegue pase por dos puntos distintos dados. 
-Dos puntos distintos pueden superponerse en una misma hoja de papel y el pliegue que se forma representa la mediatriz del segmento de recta determinado por ese par de puntos.

-Dos pliegues en una misma hoja de papel pueden superponerse. Si ambos pliegues se intersecan, su intersección es un punto solamente; determinándose dos pares de ángulos opuestos por el vértice, de modo que al hacer coincidir un pliegue con el otro, se bisecan dos de estos ángulos. Si ambos pliegues no se intersecan, al superponer un pliegue con el otro, se obtiene un tercer pliegue paralelo a los dos iniciales.

-Dados un punto $\mathrm{P}$ y un pliegue $\mathrm{L}$, puede hallarse el simétrico del punto $\mathrm{P}$ respecto al pliegue L (doblando a lo largo del pliegue L).

-Dos ángulos son congruentes, si al superponerse coinciden.

-Dos segmentos son congruentes si al superponerse coinciden.

\section{¿Qué relación existe entre dos maneras distintas de construir una figura geométrica?}

En la introducción de este trabajo, se describieron las actividades correspondientes al taller $\mathrm{n}^{\circ} 2$; por ello, en el análisis de las producciones de los participantes del curso RPG-AC, se han tenido en consideración solamente lo realizado en las actividades libres, ya que, las actividades dirigidas - centradas en la lectura activa del artículo elaborado por Arrieche \& Iglesias (2010) - se considera que permitieron ejemplificar la manera esperada de llevar a cabo la tarea: seleccionar una figura geométrica y realizar su construcción con doblado de papel y con el Cabri II, tratando de establecer la equivalencia entre ambas construcciones y presentar la debida justificación. En el cuadro 5, se mencionan las construcciones realizadas por cinco de los seis grupos de trabajo. Cabe señalar que del grupo $\mathrm{n}^{\circ} 6$ no se disponía de los recaudos necesarios para analizar sus construcciones apropiadamente; por ello, desde el punto de vista investigativo, no se consideraron sus producciones académicas.

Cuadro 5. Construcciones seleccionadas por los grupos de trabajo

\begin{tabular}{|l|l|}
\hline Grupo $\mathrm{n}^{\circ} 1$ & Construir un cuadrado, partiendo de otro cuadrado. \\
\hline Grupo $\mathrm{n}^{\circ} 2$ & Construcción de un triángulo equilátero a partir de un rectángulo. \\
\hline Grupo $\mathrm{n}^{\circ} 3$ & Construcción de un pentágono regular. \\
\hline Grupo $\mathrm{n}^{\circ} 4$ & Construcción de un hexágono, a partir un triángulo equilátero. \\
\hline Grupo $\mathrm{n}^{\circ} 5$ & Construcción de un triángulo isorrectángulo, partiendo de un cuadrado \\
\hline
\end{tabular}

Fuente: Elaboración Propia 
Para analizar las producciones de los estudiantes durante el desarrollo del taller $\mathrm{n}^{\circ} 2$, se consideraron los informes escritos (elaborados haciendo uso del editor de texto Word), los archivos fig elaborados con el Cabri II (el protocolo de construcción empleado por los estudiantes se conoce haciendo uso de la herramienta "mostrar la descripción" disponible en el Cabri II) y grabaciones de audio-video de ciertos episodios (su revisión y transcripción complementaron lo presentado por los distintos grupos de trabajo en los informes escritos y archivos .fig). Los estudiantes del curso de RPG-AC han sido identificados con las iniciales de sus nombres y apellidos (por ejemplo: BP, WR, YC, AO, ZT, OB) y lo que expresaron se ha escrito con cursiva. A modo de ilustración, seguidamente se darán a conocer las construcciones realizadas por los tres primeros grupos.

El grupo $\mathrm{n}^{\mathrm{o}} 1$ decidió trabajar con la construcción de un cuadrado a partir de otro cuadrado; en cuanto a la construcción con doblado de papel siguieron el procedimiento descrito en el cuadro 6, la cual fue elaborado con información disponible en el informe escrito presentado por dicho grupo, incluyendo las figuras ilustrativas del procedimiento empleado. 
Cuadro 6. Construcción de un cuadrado, a partir de otro cuadrado, con doblado de papel

\begin{tabular}{|c|c|}
\hline $\begin{array}{l}\text { Procedimiento } \\
\end{array}$ & Figura ilustrativa \\
\hline 1. Se tiene el cuadrado ABCD. & ${ }^{A}$ \\
\hline $\begin{array}{c}2 . \quad \text { Se hace coincidir el vértice } \mathrm{D} \text { con el } \\
\text { vértice } \mathrm{B} \text {, obteniendo así la diagonal } \mathrm{AC} \text {. }\end{array}$ & \\
\hline $\begin{array}{c}\text { 3. Se hace coincidir el vértice } \mathrm{A} \text { con el } \\
\text { vértice } \mathrm{C} \text {, obteniendo así la diagonal } \mathrm{BD} \text {. }\end{array}$ & \\
\hline $\begin{array}{l}\text { 4. Se halla el punto de intersección de las } \\
\text { diagonales, siendo este } \mathrm{O} \text {. }\end{array}$ & A \\
\hline $\begin{array}{l}\text { 5. Se hace coincidir el vértice A con } \mathrm{O} \text {, } \\
\text { determinando así los puntos medios de los lados } \\
\mathrm{AD} \text { y } \mathrm{AB} \text {, siendo estos } \mathrm{E} \text { y } \mathrm{F} \text { respectivamente, } \\
\text { constituyendo el segmento EF. }\end{array}$ & $D^{L}$ \\
\hline $\begin{array}{l}\text { 6. Se hace coincidir el vértice } \mathrm{C} \text { con } \mathrm{O} \text {, } \\
\text { determinando así los puntos medios de los lados } \\
\mathrm{BC} \text { y } \mathrm{CD} \text {, siendo estos } \mathrm{G} \text { y } \mathrm{H} \text { respectivamente, } \\
\text { constituyendo el segmento GH. }\end{array}$ & $\sum_{D}^{A}$ \\
\hline $\begin{array}{l}\text { 7. Se hace coincidir los vértices B y D (con } \\
\text { el punto O) para lograr conseguir los segmentos } \\
\text { FG y EH. De esta manera se obtiene el } \\
\text { cuadrilátero EFGH. Ahora bien, probemos que } \\
\text { este cuadrilátero es un cuadrado. }\end{array}$ & $\sum_{D}^{A}$ \\
\hline
\end{tabular}

\section{Fuente: Elaboración Propia}

Una vez realizada la construcción con doblado de papel (descrita en el cuadro 6), el grupo $\mathrm{n}^{\mathrm{o}} 1$ probó que el cuadrilátero $\mathrm{EFGH}$ (determinado por los puntos medios de los lados del cuadrado ABCD) es un cuadrado, teniendo como referencia la Figura 2:

Hagamos coincidir los puntos $B$ y $D$ con el punto $O(1)$; así $F O=O H$ y $E O=O G$, siendo $\overline{F H}$ y $\overline{E G}$ las diagonales de EFHG (2). $\overline{O F} \perp \overline{E G}$, ya que, el ángulo $\Varangle F O E$ es recto (3) $y$, por ángulos opuestos por el vértice y ángulos suplementarios, sabemos que las diagonales del cuadrilátero son perpendiculares entre si y son congruentes, ya que la longitud de las mismas es igual a la de los lados del cuadrado ABCD (4) entonces por propiedades 
(atributos) del cuadrado podemos decir que EFGH es un cuadrado (Justificación dada por el grupo $n^{\circ} 1$ ).

Figura 2. Figura empleada por el grupo $\mathrm{n}^{\circ} 1$ para probar que el cuadrilátero EFGH es un cuadrado.

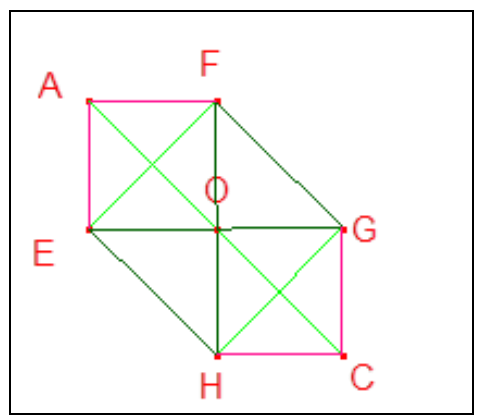

Fuente: Elaboración Propia

Figura 3. Figura auxiliar elaborada por los investigadores para ilustrar la justificación dada por el grupo $\mathrm{n}^{\mathrm{o}} 1$.

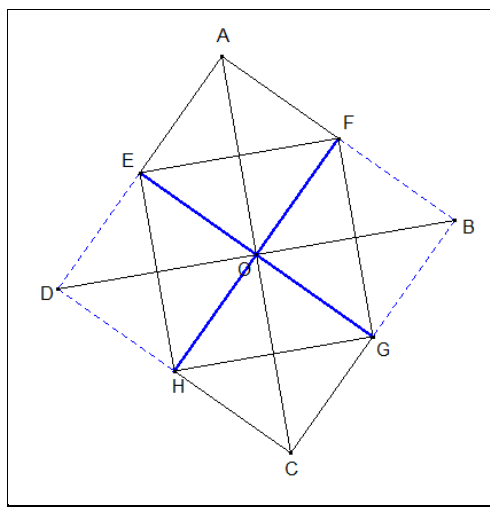

Fuente: Elaboración Propia

Nótese que el grupo $\mathrm{n}^{\mathrm{o}} 1$ inicia la prueba (ver figuras 2 y 3), haciendo coincidir los vértices $\mathrm{B}$ y $\mathrm{D}$ del cuadrado original con el punto $\mathrm{O}(1)$; de este modo, el segmento $\overline{D E}$ se superpone con el segmento $\overline{O E}$ y el segmento $\overline{D H}$ con el segmento $\overline{O H}$ (DE $=\mathrm{OE}$ y $\mathrm{DH}=$ $\mathrm{OH})$. De manera similar, el segmento $\overline{B F}$ se superpone con el segmento $\overline{O F}$ y el segmento $\overline{B G}$ con el segmento $\overline{O G}(\mathrm{BF}=\mathrm{OF}$ y $\mathrm{BG}=\mathrm{OG})$. Además, los segmentos $\overline{O E}, \overline{O H}, \overline{O F}$ y $\overline{O G}$ son congruentes entre sí, ya que, su longitud es L/2, donde L es la longitud de los lados del cuadrado ABCD; esto es lo que le permite afirmar en (2) que las diagonales $\overline{E G}$ y $\overline{F H}$ del cuadrilátero EFGH se bisecan en O. Asimismo, afirman, en (3), que < FOE es recto (lo cual es cierto), pero teniendo en cuenta que parten del coincidir los puntos $\mathrm{B}$ y D con el punto $\mathrm{O}$, debieron primero establecer que < FOG es recto, ya que, se superpone con el ángulo recto < FBG y, por ello, también son ángulos rectos: < FOE (por ser el suplemento de un ángulo recto) y $<\mathrm{EOH}$ (por ángulos opuestos por el vértice). Hasta aquí han probado que las diagonales del cuadrilátero EFGH se bisecan y son perpendiculares entre sí; luego afirman en (4) que son congruentes, ya que tienen la misma longitud de los lados del cuadrado ABCD. 
Pudiera decirse, como se ha tratado de ilustrar en este comentario, que al trabajar con el doblado de papel, asumieron - aunque no lo hayan mencionado explícitamente - las relaciones existentes entre segmentos y ángulos al hacer coincidir un punto con otro, porque disponían de evidencias suficientes.

Cuadro 7. Construcción de un cuadrado, a partir de otro cuadrado, en un AGD

\begin{tabular}{|c|c|c|}
\hline Procedimiento & \multicolumn{2}{|c|}{ Herramientas empleadas } \\
\hline 1. Se traza el segmento $\overline{D C}$. & \multirow{3}{*}{\multicolumn{2}{|c|}{$\begin{array}{l}\text { Punto } \\
\text { Sistema de coordenadas: _- } \\
\text { Punto } \\
\text { Punto } \\
\text { Punto (Vértice de un polígono regular): _, C } \\
\text { Punto (Vértice de un polígono regular): D } \\
\text { Punto (Vértice de un polígono regular): A } \\
\text { Polígono regular (Polígono regular): B, C, D, A }\end{array}$}} \\
\hline $\begin{array}{l}\text { 2. Se traza una perpendicular }\left(\mathbf{L}_{1}\right) \text { (al } \\
\text { segmento } \overline{\boldsymbol{D C}}) \text { en el punto } \mathrm{C} \text { y }\left(\mathrm{L}_{2}\right) \text { en el } \\
\text { punto } D \text { respectivamente. }\end{array}$ & & \\
\hline $\begin{array}{l}\text { 3. Con centro en } \mathrm{C} \text { y radio en } \overline{C D} \text { se } \\
\text { traza un arco de circunferencia. Con centro } \\
\text { en } \mathrm{D} \text { y radio DC, se traza un arco de } \\
\text { circunferencia. }\end{array}$ & & \\
\hline $\begin{array}{l}\text { 4. Se hallan los puntos de intersección } \\
\text { de } \mathrm{C}_{1} \text { y } \mathrm{C}\left(\mathbf{L}_{1}\right) ; \mathrm{C}_{2} \text { y } \mathrm{D}\left(\mathbf{L}_{2}\right) \text {, encontrando así } \\
\text { los puntos A y B. }\end{array}$ & \multirow{4}{*}{\multicolumn{2}{|c|}{$\begin{array}{l}\text { Observación } n^{\circ} \text { 1: Con el Cabri II, construyeron el cuadrado } \\
\text { BCDA, haciendo uso de la herramienta polígono regular de } \mathrm{n} \\
\text { lados (en este caso, } \mathrm{n}=4 \text { ); sin embargo, en la descripción de los } \\
\text { pasos a seguir, parten del trazado del segmento } \overline{D C} \text {. }\end{array}$}} \\
\hline $\begin{array}{c}\text { 5. Se trazan los segmentos } \overline{A B}, \overline{B C} \text { y } \\
\overline{A D} ; \text { obteniendo así un cuadrado } \mathbf{A B C D} \text {. }\end{array}$ & & \\
\hline $\begin{array}{l}\text { 6. Se trazan los segmentos } \overline{A C} \text { y } \overline{B D} \\
\text { siendo estas las diagonales del cuadrado } \\
\text { ABCD. }\end{array}$ & & \\
\hline $\begin{array}{l}\text { 7. Se halla el punto de intersección } \\
\text { entre las diagonales, denominándolo } \mathrm{O} \text {. }\end{array}$ & & \\
\hline $\begin{array}{l}\text { 8. Se halla el punto medio de los } \\
\text { segmentos } \overline{A D}, \overline{A B}, \overline{B C} \text { y } \overline{C D} \text { siendo los } \\
\text { mismos E, F, G y H respectivamente. }\end{array}$ & \multicolumn{2}{|l|}{$\begin{array}{l}\text { F Punto (Punto medio): } \\
\text { E Punto (Punto medio): } \\
\text { H Punto (Punto medio): } \\
\text { G Punto (Punto medio): }\end{array}$} \\
\hline $\begin{array}{l}\text { 9. Se trazan los } \text { segmentos } \overline{F G}, \\
\overline{G H}, \overline{H E} \text { y } \overline{E F} .\end{array}$ & $\begin{array}{l}\text { Segmento: E, F } \\
\text { Segmento: F, G }\end{array}$ & $\begin{array}{l}\text { Segmento: } \mathrm{G}, \mathrm{H} \\
\text { Segmento: } \mathrm{H}, \mathrm{E}\end{array}$ \\
\hline $\begin{array}{l}\text { 10. Se considera la recta } \overleftrightarrow{F G} \text { y se realiza } \\
\text { la simetría axial del triángulo FBG con } \\
\text { respecto a la recta. }\end{array}$ & $\begin{array}{l}\text { Triángulo: E, H, D } \\
\text { Triángulo: G, H, C } \\
\text { Triángulo: G, B, F } \\
\text { Triángulo: F, E, A } \\
\text { Recta: F, G } \\
\text { Recta: G, H }\end{array}$ & $\begin{array}{l}\text { Recta: H, E } \\
\text { Recta: E, F } \\
\text { Punto (Simetría axial): F, } \\
\text { Punto (Simetría axial): E, } \\
\text { Punto (Simetría axial): A, } \\
\text { Triángulo:_,___ }\end{array}$ \\
\hline 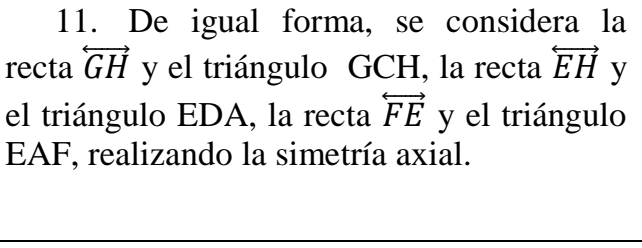 & $\begin{array}{l}\text { Punto (Simetría axial): } \mathrm{E},{ }_{-} \\
\text {Punto (Simetría axial): } \mathrm{H}_{-} \\
\text {Punto (Simetría axial): } \mathrm{D}{ }_{-} \\
\text {Triángulo: _, } \\
\text { Punto (Simetría axial): } \mathrm{G},{ }_{-} \\
\text {Punto (Simetría axial): } \mathrm{H}_{-}\end{array}$ & $\begin{array}{l}\text { Punto (Simetría axial): } \mathrm{C}_{-} \\
\text {Triángulo: _,_, } \\
\text { Punto (Simetría axial): } \mathrm{G},{ }_{-} \\
\text {Punto (Simetría axial): } \mathrm{B},{ }_{-} \\
\text {Punto (Simetría axial): } \mathrm{F}{ }_{-} \\
\text {Triángulo:__,_,_ }\end{array}$ \\
\hline \multicolumn{3}{|c|}{$\begin{array}{l}\text { Observación } n^{\circ} 2: \\
\text { A partir del paso } 8 \text {, hay correspondencia entre lo descrito y la construcción realizada con el Cabri II; ubican el } \\
\text { punto medio de cada uno de los lados del cuadrado BCDA. Trazan los segmentos determinados por los puntos } \\
\text { medios de los lados del cuadrado BCDA y construyen los triángulos EHD, GHC, GBF y FEA y trazan las rectas } \\
\overleftrightarrow{F G}, \overrightarrow{G H}, \overrightarrow{H E} \text { y } \overrightarrow{E F} \text {. Luego, buscan la imagen simétrica del triángulo FEA con respecto a la recta } \overrightarrow{F E} \text {. } \\
\text { Obsérvese que, a pesar que, construyeron el triángulo FEA, no le aplicaron la herramienta simetría axial, sino } \\
\text { que se la aplicaron a cada uno de los vértices (como lo harían si estuvieran trabajando manualmente). } \\
\text { De manera análoga, determinan la imagen simétrica de los triángulos EHD, GHC y GBF con respecto a las rectas } \\
\overleftrightarrow{E D}, \overleftrightarrow{G H} \text { y } \overleftrightarrow{F G} \text {. Nótese que la descripción no coincide con el orden seguido en la construcción, aunque esto no } \\
\text { alteraría lo realizado }\end{array}$} \\
\hline
\end{tabular}


En el Cuadro 7, se presenta el procedimiento descrito por el grupo $\mathrm{n}^{\mathbf{0}} 1$ y las herramientas empleadas para construir un cuadrado, a partir de otro cuadrado, en un ambiente de Geometría Dinámica (AGD); en el cual, el hallar la imagen simétrica de una figura con respecto a un eje equivale a doblar el papel a través de ese eje de simetría.

El grupo n ${ }^{\circ} 1$ mostró la justificación siguiente: De esta manera se obtiene el polígono $F E H G$, el cual es un cuadrado por $G O=O E$ y $F O=O H ; Y$ ellos son congruentes entre sí, ya que ellos corresponden a 1/2 del lado del cuadrado $A B C D(1) . \overleftrightarrow{F H}$ y $\overleftrightarrow{E G}$ sonlas diagonales del polígono, las mismas son congruentes y ortogonales entre sí. Son ortogonales porque forman un ángulo de 90, ya que, el ángulo FOE se corresponde con el ángulo EAF por la simetría axial. De esta forma se ha demostrado que el polígono EFGH es un cuadrado (2).

Se observa en la afirmación (1) que demuestran que las diagonales del cuadrilátero EFGH se bisecan en el punto $\mathrm{O}$, teniendo en cuenta que los segmentos $\overline{E O}, \overline{F O}, \overline{G O}$ y $\overline{H O}$ son congruentes entre sí, porque su longitud es igual a la mitad de la longitud de los lados del cuadrado original; sin embargo, no lo señalan de forma explícita. Esto es clave porque las diagonales de un cuadrilátero pueden ser congruentes y perpendiculares, sin llegar a bisecarse y el cuadrilátero no sería un cuadrado. En (2), aplican que un triángulo y su imagen simetría son congruentes entre sí; y, por partes correspondientes de triángulos congruentes (PCTC), garantizan que el < FOE es un ángulo recto como lo es < FAE.

Al revisar las justificaciones dadas por el grupo $n^{\mathbf{0}} 1$, con el propósito de garantizar que el cuadrilátero EFGH es un cuadrado, se nota que siguieron una idea central: demostrar que sus diagonales $\overline{E G}$ y $\overline{F H}$ se bisecan en el punto $\mathrm{O} \mathrm{y}$, además, son congruentes $\mathrm{y}$ perpendiculares entre sí; aquí se estarían basando en ciertas propiedades estudiadas en el curso de Geometría I: (a) Un cuadrado es un rombo y un rectángulo; (b) Si las diagonales de un cuadrilátero se bisecan y son perpendiculares, entonces el cuadrilátero es un rombo; (c) Si las diagonales de un cuadrilátero se bisecan y son congruentes, entonces el cuadrilátero es un rectángulo. En función de la construcción realizada y los datos disponibles, se considera que pudo probar que los triángulos EHD, GHC, GBF y FEA son congruentes entre sí y que los lados $\overline{E H}, \overline{G H}, \overline{G F}$ y $\overline{F E}$ son congruentes, con lo cual pueden llegar a establecer que el cuadrilátero EFHG es un paralelogramo con lados congruentes entre sí (un rombo); luego tendrían que probar que posee un ángulo recto. 
El grupo $n^{\circ} 2$ seleccionó la construcción de un triángulo equilátero, partiendo de una hoja DIN A4, mediante el doblado de papel. Cabe señalar que, para reconstruir el procedimiento empleado, se revisó el archivo de audio y video disponible, el cual fue grabado durante el desarrollo de la actividad. En el cuadro 8, se ha transcrito el procedimiento empleado por el grupo $\mathrm{n}^{\mathrm{o}} 2$ y se ha acompañado de una figura ilustrativa para facilitar su seguimiento por parte de los lectores y algunas notas realizadas por los investigadores.

Cuadro 8. Procedimiento empleado para construir un triángulo equilátero con doblado de papel

\begin{tabular}{|c|c|}
\hline $\begin{array}{l}\text { Procedimiento } \\
\end{array}$ & Figuras ilustrativas \\
\hline $\begin{array}{l}\text { HB: Vamos a construir un triángulo equilátero con } \\
\text { doblado de papel, partiendo de una hoja conocida } \\
\text { como DIN A4. } \\
\text { En una hoja DIN A4, la razón entre las longitudes } \\
\text { de dos lados consecutivos es raíz cuadrada de } 2 \text {. }\end{array}$ & \\
\hline $\begin{array}{l}\text { HB: El primer paso que vamos hacer es unir dos } \\
\text { vértices los más cercanos, de modo que } \\
\text { encontremos los puntos medios de esos lados } \\
\text { paralelos; vemos que se forma una línea, que nos } \\
\text { representaría un segmento, que en este nos daría } \\
\text { un eje de simetría, por formar dos rectángulos } \\
\text { congruentes. } \\
\text { En el rectángulo ABCD, se hace coincidir el } \\
\text { vértice A con el B y el vértice } \mathrm{D} \text { con el C, } \\
\text { obteniendo los puntos medios W y X de los lados } \\
\overline{A B} \text { y } \overline{D C} \text { respectivamente. }\end{array}$ & \\
\hline $\begin{array}{l}\text { HB: Ahora bien colocamos la hoja en forma } \\
\text { horizontal. El vértice superior izquierdo lo vamos } \\
\text { a trasladar de manera tal que el vértice quede } \\
\text { sobre el eje de simetría hallado y a su vez que } \\
\text { biseque (al ángulo con vértice en) el vértice } \\
\text { inferior izquierdo. } \\
\text { Se hace coincidir el vértice A con un punto A' del } \\
\text { segmento } \overline{W X} \text {, de modo tal que el segmento } \overline{B E} \\
\text { biseque al }<\mathrm{ABA} \text {. En la descripción se nota } \\
\text { ciertas imprecisiones en el uso del lenguaje. }\end{array}$ & $\mathrm{x}$ \\
\hline $\begin{array}{l}\text { HB: Luego de tener este doblado vamos hacer un } \\
\text { doblez tomando en cuenta la parte que nos queda } \\
\text { en el interior del rectángulo, hacemos un doblez } \\
\text { del ángulo contrario a través de esa línea, } \\
\text { tratando de prolongarla y que nos quede de forma } \\
\text { recta; puede ser hacia dentro o hacia fuera (se } \\
\text { puede trabajar cualquiera de los dos vértices). El } \\
\text { vértice tiene que coincidir con el lado } \\
\text { Teniendo plegado el triángulo ABE sobre el } \\
\text { triángulo A'BE, se hace coincidir el vértice E con } \\
\text { un punto F del lado } \overline{B C} \text {; de esta manera, BE = BF. }\end{array}$ & $i^{\prime}$ \\
\hline $\begin{array}{l}\text { HB: Luego en este punto en la parte inferior, 'nos } \\
\text { tuvo que sobrar un triángulo pequeño', este lo } \\
\text { vamos a doblar hacia arriba; y de esta manera } \\
\text { conseguimos el triángulo equilátero. }\end{array}$ & $\begin{array}{l}\text { Esto posiblemente sucedió porque la } \\
\text { construcción no la realizaron con una hoja } \\
\text { DINA4, sino con una hoja tamaño carta. }\end{array}$ \\
\hline
\end{tabular}


Una vez que HB realizó la construcción con doblado de papel de un triángulo equilátero, otro integrante del grupo WR preguntó: Ahora ¿cómo sabemos que es un triángulo equilátero? Seguidamente dijo: Buscamos las medianas de cada lado del triángulo, procediendo - de inmediato - a efectuar los correspondientes dobleces; es decir, hace coincidir el vértice B con $\mathrm{F}$, obteniendo la mediana EE', siendo $\mathrm{E}$ ' el punto medio del segmento $\overline{B F} \mathrm{y}$, en forma análoga, construye las medianas $\overline{F F^{\prime}}$ y $\overline{B B^{\prime}}$ como se ilustra en la figura 4 :

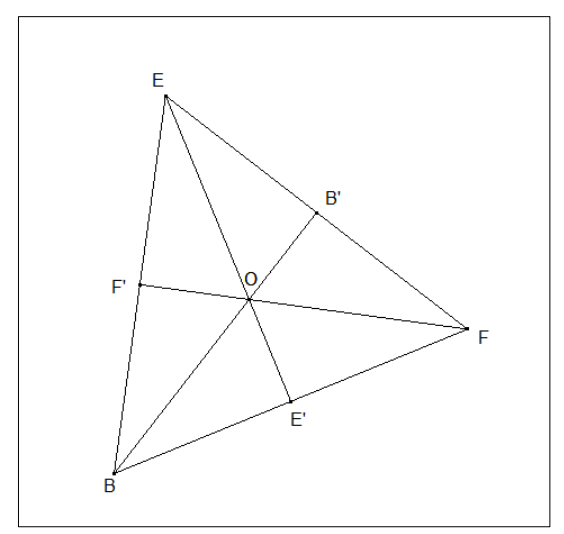

Figura 4. Figura ilustrativa de las medianas de un triángulo equilátero

WR, una vez realizados los dobleces, afirma: Encontrando así el punto de intersección de todas las medianas. ¿Qué se quiere demostrar con esto? Que todos los triángulos que se forman son congruentes.

Resulta interesante que la explicación dada por el grupo $n^{\circ} 2$ esté basada en el hecho que dos figuras son congruentes si al superponerse coinciden y, además que, si las medianas de un triángulo lo descomponen en seis triángulos congruentes, entonces el triángulo es equilátero. En la figura y, por superposición, WR llegó a que los triángulos $\Delta \mathrm{OBE}^{\prime}, \Delta \mathrm{OFE}$ ', $\Delta$ OFB', $\Delta$ OEB', $\triangle$ OEF' y $\Delta$ OBF' son congruentes entre sí; dejando implícito que, de esta manera, los segmentos $\overline{B E}, \overline{F E}, \overline{F B^{\prime}}, \overline{E B^{\prime}}, \overline{E F^{\prime}}$ y $\overline{B F^{\prime}}$ son congruentes entre sí, por partes correspondientes de triángulos congruentes $(\mathrm{PCTC}) \mathrm{y}$, en consecuencia, $\mathrm{BF}=\mathrm{FE}=\mathrm{EB}$.

Cabe señalar que, aparentemente al hacer la construcción del triángulo BEF con doblado de papel, los integrantes del grupo $\mathrm{n}^{\mathrm{o}} 2$, no se percataron que, por superposición, los ángulos $\mathrm{ABE}, \mathrm{A}^{\prime} \mathrm{BE}$ y $\mathrm{A}^{\prime} \mathrm{BF}$ son congruentes y, por ende, se ha trisecado el ángulo recto $\mathrm{ABC}$ y, por el postulado de adición de ángulos, el ángulo $\mathrm{EBF}$ mide $60^{\circ}$ con $\mathrm{BE}=\mathrm{BF}$; con estas premisas, es posible probar que el triángulo $\mathrm{BEF}$ es equilátero (probando que $\Delta \mathrm{BEF}$ es 
equiángulo). Se conoce que los ángulos en la base de un triángulo isósceles son congruentes (y hasta aquí se conoce que el triángulo BEF es isósceles) y, por ello, los ángulos BEF y BFE son congruentes $\mathrm{y}$, además, $\mathrm{m}(<\mathrm{BEF})+\mathrm{m}(<\mathrm{BFE})=120^{\circ}$, ya que la suma de los ángulos internos de un triángulo es igual a $180^{\circ}$ y el ángulo restante mide $60^{\circ}$. Esta situación fue aprovechada por la facilitadora para propiciar la participación de los profesores en formación y el intercambio de ideas durante el desarrollo de la exposición realizada por el grupo $\mathrm{n}^{\circ} 2$.

Para finalizar su intervención, los participantes del grupo $\mathrm{n}^{\mathrm{o}} 2$, usando la opción "revisar la construcción" disponible en el Cabri II, describieron el procedimiento utilizado para construir un triángulo equilátero $\mathrm{BEF}$, a partir de un rectángulo $\mathrm{ABCD}$, en el cual la razón entre las longitudes de dos lados consecutivos es raíz cuadrada de 2. Se observa, en el cuadro 9, que procuraron establecer relaciones con el procedimiento utilizado con el doblado de papel; sin embargo, al iniciar la construcción con el Cabri II, no construyen un rectángulo que satisfaga las condiciones exigidas. Además, expresan que la clave está en la trisección de un ángulo recto; sin embargo, la manera de hacerlo es inconsistente (ver paso 3 en el mencionado cuadro). Cabe decir que la manera de trisecar - con regla y compás - un ángulo recto lo trataron Arrieche \& Iglesias (2010) y la misma fue utilizada por los participantes en las actividades dirigidas. En el cuadro 9, se han incorporado una serie de observaciones como parte del análisis de esta actividad. Es necesario señalar que los integrantes del grupo $n^{\circ} 2$, en función a las observaciones realizadas durante el desarrollo de la exposición, asumieron los errores cometidos (inconsistencia de la trisección de un ángulo recto y el haber partido de cualquier rectángulo). Quedándole planteada una tarea: ¿Cómo construir un rectángulo en el cual la razón entre las longitudes de dos lados consecutivos es raíz cuadrada de 2 ? 
Cuadro 9. Construcción de un triángulo equilátero, a partir de un rectángulo, en un AGD

\begin{tabular}{|c|c|}
\hline Procedimiento & Herramientas empleadas \\
\hline $\begin{array}{l}\text { HB: (1) Ahora realizaremos la misma construcción, } \\
\text { pero esta vez utilizando el Cabri. Esta primera parte } \\
\text { es para construir el rectángulo cuya razón es raíz de } \\
\text { dos. }\end{array}$ & $\begin{array}{ll} & \text { Punto } \\
& \text { Sistema de coordenadas: } \\
\text { B } & \text { Punto } \\
\text { C } \quad \text { Punto } \\
\\
\text { Segmento: B, C } \\
\text { Recta (Recta perpendicular): B, } \\
\text { Recta (Recta perpendicular): C, } \\
\text { A } \quad \begin{array}{l}\text { Punto (Punto sobre un objeto): } \\
\text { Recta (Recta paralela): A, }\end{array} \\
\text { D } \quad \begin{array}{l}\text { Punto (Punto(s) de intersección): _, } \\
\text { Polígono: A, D, C, B }\end{array}\end{array}$ \\
\hline $\begin{array}{l}Y C:(2) \text { Tenemos los puntos } A, B, C \text { y } D \text {, los unimos } \\
\text { determinando el rectángulo. Luego determinamos los } \\
\text { puntos medios de (los lados) } \overline{A B} \text { y } \overline{C D} \text { y unimos } \\
\text { ambos puntos, determinado el segmento } \overline{W X} \text {. } \\
\text { HB: De manera de asemejar los pasos que realizamos } \\
\text { con doblado de papel al uso del software. Así este } \\
\text { segmento } \overline{W X} \text { es la línea que se ve cuando hacemos el } \\
\text { doblado de papel, lo mismo que el eje de simetría. }\end{array}$ & $\begin{array}{ll}\text { W } & \text { Punto (Punto medio): A, B } \\
& \text { Punto (Punto sobre un objeto): } \\
& \text { Punto (Punto medio): D, } \\
\text { X } & \text { Punto (Punto medio): D, C } \\
& \text { Segmento: W, X }\end{array}$ \\
\hline $\begin{array}{l}\text { HB: (3) Ahora como sabemos que un ángulo recto lo } \\
\text { podemos trisecar, nos basaremos en esto para tratar } \\
\text { de hacer la construcción. Por lo que trisecaremos el } \\
\text { ángulo recto } \angle A B C . \text { De esta manera se nota que la } \\
\text { semirrecta } \stackrel{B}{B} \text { interseca al } \overline{W X} \text { en un punto } A^{\prime} \text {. } \\
\text { Trazamos la semirrecta } \overrightarrow{B 2} \text { e interseca al } \overline{A D} \text { en un } \\
\text { punto E. }\end{array}$ & $\begin{array}{ll}\text { P } & \text { Punto (Punto sobre un objeto): WX } \\
& \text { ángulo: A, B, P } \\
& \text { Semirrecta: B, P } \\
& \text { Recta (Bisectriz): W, B, P } \\
\text { E } & \text { Punto (Punto(s) de intersección): _, } \\
& \text { Punto (Punto(s) de intersección): _, } \\
& \text { Punto (Punto(s) de intersección): _, } \\
& \text { Triángulo: A, B, E } \\
\text { A } \quad \text { Punto (Simetría axial): A, } & \\
& \text { Punto (Simetría axial): B, } \\
& \text { Punto (Simetría axial): E, } \\
& \text { Triángulo: A , _, } \\
\end{array}$ \\
\hline $\begin{array}{l}\text { HB: Lo próximo que se hace es marcar el triángulo } \\
\text { ABE y aplicamos simetría axial con respecto a esa } \\
\text { semirrecta (que sería el segundo doblado que } \\
\text { trabajamos), como se puede notar el vértice A } \\
\text { coincide con el (lado) WX. Ahora trazamos una } \\
\text { semirrecta que parte del punto E y pasa por el punto } \\
\text { A', intersecando al (segmento) } \overline{A C} \text { en un punto F. } \\
\text { ¿Para qué hacemos esto? Para hacer que en este } \\
\text { polígono EDCF a través de simetría axial con } \\
\text { respecto a esa semirrecta } \overline{E A} \text { que acabamos de trazar } \\
\text { nos pueda representar como el próximo doblado de } \\
\text { papel que trabajamos. } \\
\text { Por lo que la punta que sobra en la pantalla es la } \\
\text { punta que sobra en el doblado de papel. Ese triángulo } \\
\text { como lo hicimos anteriormente lo doblamos, en este } \\
\text { caso vamos a marcar el triángulo y por simetría axial } \\
\text { con respecto al segmento CD tenemos todos los } \\
\text { doblados y nos quedaría un triángulo equilátero } \\
\Delta B E F \text {. }\end{array}$ & $\begin{array}{l}\text { Semirrecta: E, A } \\
\text { F Punto (Punto(s) de intersección): _, } \\
\text { Polígono: E, D, C, F } \\
\text { Punto (Simetría axial): E, } \\
\text { Punto (Simetría axial): D, } \\
\text { Punto (Simetría axial): C, } \\
\text { Punto (Simetría axial): F, } \\
\text { Polígono: _,_,_,_- } \\
\text { Punto (Punto(s) de intersección): _, - } \\
\text { Triángulo:_, F, _- } \\
\text { Punto (Simetría axial): _, _- } \\
\text { Punto (Simetría axial): F, _- } \\
\text { Punto (Simetría axial): _, _- } \\
\text { Triángulo: _,_, _- } \\
\text { Triángulo: B, E, F } \\
5,11 \mathrm{~cm} \text { Texto (Distancia o longitud): E, B } \\
5,11 \mathrm{~cm} \text { Texto (Distancia o longitud): B, F } \\
5,11 \mathrm{~cm} \text { Texto (Distancia o longitud): F, E }\end{array}$ \\
\hline
\end{tabular}


Nótese que, en el paso 3, ubican un punto $\mathrm{P}$ sobre el segmento $\overline{W X}$ y marcan el ángulo ABP y trazan la semirrecta $\overrightarrow{B P}$; esto no garantiza que la $\mathrm{m}(<\mathrm{ABP})=60^{\circ}$. Luego trazan la bisectriz del < WBP, la cual interseca al lado $\overline{A D}$ en el punto E. Trazan el triángulo ABE y buscan su imagen simétrica con respecto a la recta $\overleftrightarrow{B E}$. Así, hallan un punto $A^{\prime}$ imagen simétrica de A con respecto a la recta $\overleftrightarrow{B E}$, el cual no necesariamente pertenece al segmento $\overline{W X}$. No obstante, arrastrando el punto $\mathrm{P}$, puede lograrse que el punto $\mathrm{A}^{\prime}$ esté sobre el segmento $\overline{W X}$; esto sucede cuándo el ángulo $\mathrm{ABP}$ mide $60^{\circ}$. En la figura 5, el punto A' no coincide con el punto $\mathrm{P}$ y el triángulo BEF no es equilátero. Aquí se observa una trisección inconsistente de un ángulo recto. Luego trazan la semirrecta $\overrightarrow{E A}$ que corta al lado $\overline{B C}$ en un punto F. Tal semirrecta servirá como eje de simetría del polígono EDCF; así como el lado $\overline{B C}$ servirá como eje de simetría del triángulo C'FQ, siendo $\mathrm{Q}$ el punto donde el segmento C'D' corta al lado $\overline{B C}$. Con esto simulan el doblado de papel.

La clave de la construcción está en la trisección de un ángulo recto; en este caso, el ángulo $\mathrm{ABC}$, ya que así el triángulo $\mathrm{ABE}$ es un triángulo $30^{\circ}-60^{\circ}-90^{\circ}$ y también lo será su imagen simétrica: $\Delta$ A'BE, con lo cual $\angle \mathrm{A}^{\prime} \mathrm{EB}$ mide $60^{\circ}$. De esta manera, el triángulo $\mathrm{BEF}$ tendrá dos ángulos de $60^{\circ}$, con lo cual el ángulo EFB también mide $60^{\circ}$. Por ende, si el triángulo BEF es equiángulo también es equilátero. Nótese que se pudiera conjeturar que, siguiendo la construcción si el < ABP no mide $60^{\circ}$, el triángulo $\mathrm{BEF}$ seria isósceles con $\mathrm{BF}=\mathrm{EF}$. ¿Por qué?

Figura 5. Trisección inconsistente de un ángulo recto

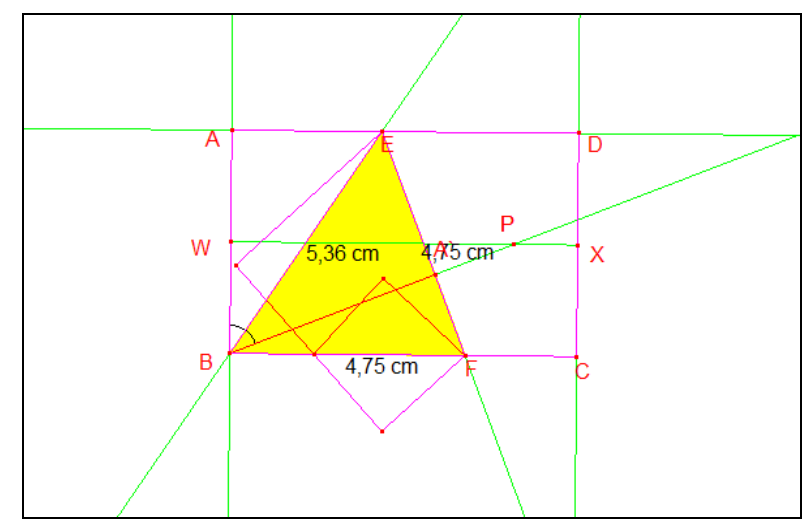

El grupo $n^{\circ} 3$ eligió trabajar con la construcción de un pentágono regular, a partir de una hoja DIN A4 (o una hoja rectangular que cumple la condición de que la longitud de la base $b$ entre la longitud de la altura $h$ sea $\sqrt{ } 2$ ). Cabe señalar que, en Venezuela, no es habitual conseguir este tipo de hoja, usualmente se trabaja con una hoja de papel tamaño carta $(21,59$ cm x 27,94 cm) o tamaño oficio $(21,59 \mathrm{~cm}$ x 35, $56 \mathrm{~cm})$. Inicialmente, el grupo $\mathrm{n}^{\circ} 3$ utilizó una hoja tamaño carta, lo cual implicó que el pentágono construido no fuera regular. Esto 
llevó a los integrantes del grupo $\mathrm{n}^{\circ} 3$ a preguntarse: ¿Cómo construir un rectángulo que sea semejante a una hoja DINA4? Esto representó la ejecución de una tarea preliminar. En el cuadro 10, se describe el procedimiento, con doblado de papel, utilizado por el grupo $\mathrm{n}^{\circ} 3$.

Cuadro 10. Construcción de un pentágono regular, usando una hoja DINA4

\begin{tabular}{|c|c|}
\hline Procedimiento & Figuras ilustrativas \\
\hline $\begin{array}{l}\text { 1. Se inicia la construcción a partir } \\
\text { de un rectángulo de papel que cumpla la } \\
\text { condición de que la base entre la altura sea } \\
\sqrt{ } 2 \text { (hoja DINA4). }\end{array}$ & 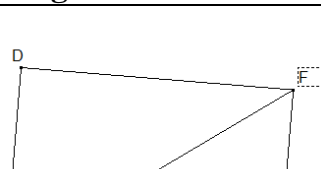 \\
\hline $\begin{array}{l}\text { 2. Trace una de las diagonales del } \\
\text { rectángulo y asígnele letras a todos los } \\
\text { vértices. }\end{array}$ & \\
\hline $\begin{array}{l}\text { Nota: A los vértices le asignaron las letras } \\
\text { ADFE }\end{array}$ & \\
\hline 3. $\quad$ Doble el papel por la diagonal. & \\
\hline $\begin{array}{l}\text { Nota: Doblaron la hoja de papel, marcando } \\
\text { el pliegue que pasa por los puntos A y F. }\end{array}$ & \\
\hline $\begin{array}{l}\text { 4. Tome los dos vértices sobrantes del } \\
\text { doblez (D y E) respectivamente, y dóblelo } \\
\text { de tal manera que se amolden a los lados } \\
\text { del rectángulo formal. }\end{array}$ & \\
\hline $\begin{array}{l}\text { Nota: En el } \triangle \mathrm{AFE} \text { ', hallaron el simétrico } \\
\text { del punto E' con respecto al lado DF; } \\
\text { obteniendo } \Delta \mathrm{E} \text { ' 'FG, donde G es el punto de } \\
\text { intersección de los segmentos DF y AE'. } \\
\text { Análogamente, hallaron la imagen simétrica } \\
\text { del punto D con respecto al segmento AE'. } \\
\text { El vocabulario empleado es impreciso; sin } \\
\text { embargo, la realización de la construcción } \\
\text { en la medida que se explicaba el } \\
\text { procedimiento permitió seguirla. }\end{array}$ & \\
\hline 5. $\quad$ Doble el vértice E al lado opuesto. & \\
\hline $\begin{array}{l}\text { 6. Finalmente tome el vértice A y } \\
\text { dóblelo, de tal manera, que el vértice toque } \\
\text { un punto del lado opuesto en el punto de } \\
\text { doblado superior realizado anteriormente. } \\
\text { Obteniéndose así EL PENTÁGONO. }\end{array}$ & \\
\hline $\begin{array}{l}\text { Nota: En el paso (5) se refieren al vértice } F \text {. } \\
\text { En } \triangle \text { AFG, A' está en el lado opuesto al } \\
\text { vértice A y F' en el lado opuesto al vértice } \\
\text { F. Se observa que } A F \text { ' = A'F', así como A'F } \\
=A^{\prime} F \text { '. }\end{array}$ & \\
\hline
\end{tabular}


Cabe señalar que las notas y figuras ilustrativas incorporadas en el cuadro 9 fue posible realizarlas mediante la observación del correspondiente audio - video; a continuación se transcribe el apartado considerado:

AO: Ahora, luego de trazar una de sus diagonales, se le va a asignar letras a cada uno de los vértices. En este caso, A (vértice inferior izquierdo), E (vértice inferior derecho), $F$ (vértice superior derecho), $D$ (vértice superior izquierdo) (Paso 2). Luego, doblar el papel por la diagonal (Paso 3). Seguidamente vamos a tomar los vértices sobrantes, en este caso E y D, doblarlos hacia cualquiera de los dos lados, pero los dos hacia la misma cara (Paso 4). Me explico, los triángulos que sobran se van a doblar hacia adentro quedándome los dos triángulos pequeños ( $\triangle E^{\prime} F G$ y $\Delta A D G$ ) sobre el triángulo mayor $(\triangle A F G)$. Esto, de tal manera que se amolden a los lados del triángulo original. Luego, el vértice $F$ se va a llevar al lado opuesto que sería el segmento AG (en el $\triangle A F G$ ) (Pasos 5 y 6).

ZT: ¿Cómo? ¡No entiendo! ¿Cómo lo coloco?

AO: Cuando nosotros tenemos el triángulo que lo superpusimos. Lo que van a hacer es lo siguiente, cualquiera de los dos vértices (de la base del triángulo) lo van a llevar al lado opuesto de manera que toque un punto del lado opuesto.

OB: ¿Cualquiera?

AO: Sí, pero de manera de que el otro vértice cuando lo doblen quede también a la misma distancia (es decir que, $A F^{\prime}=A^{\prime} F^{\prime}$ y $A^{\prime} F=A^{\prime} F^{\prime}$ ). ¿Qué pasa? Yo voy a llevar el vértice al lado opuesto de manera de que sea la diagonal o que sea la base del triángulo pequeño (lo señala en el video). Y se va a llevar, finalmente, el otro vértice que toque el punto que se realizó anteriormente en el doblez anterior, formándome así el pentágono

(Diálogo de profesores en formación durante la implementación del taller, 2018)

Para garantizar que el pentágono A'GF'BC es un pentágono regular, procedieron a trazar la mediatriz de cada uno de los lados (mediante doblado de papel) para ir verificando que los lados son congruentes entre sí, ya que, al superponerse, coinciden (ver figura 6); así, si se considera la mediatriz M1 - mediatriz del lado BC - se tiene que GF $=G^{\prime}$ y F'B = A'C y así sucesivamente.

Figura 6. Construcción de las mediatrices de los lados de un pentágono, para verificar, con doblado de papel, que los lados son congruentes entre sí.

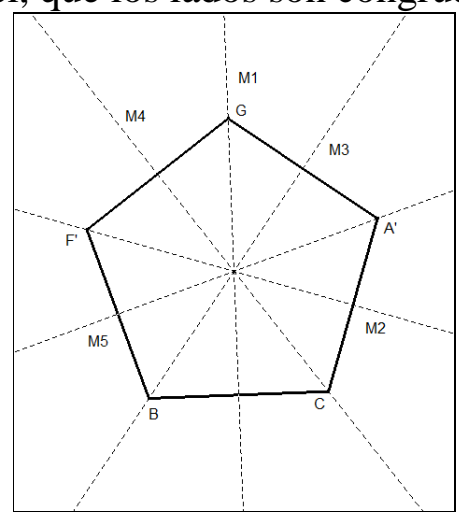


Además, como parte de las exigencias del procedimiento empleado, se vieron en la necesidad de revisar cómo dividir un segmento, de modo que las longitudes de los dos segmentos resultantes satisfagan la razón áurea (figura 7), como se muestra en el cuadro 11 (construcción auxiliar). Esto es lo que les permitió con regla y compás dividir los segmentos $\overline{A G}$ y $\overline{F G}$, hallando los puntos $\mathrm{A}^{\prime}$ y F' en los lados FG y AG respectivamente.

Figura 7. División de un segmento en dos segmentos cuyas longitudes están en razón áurea.

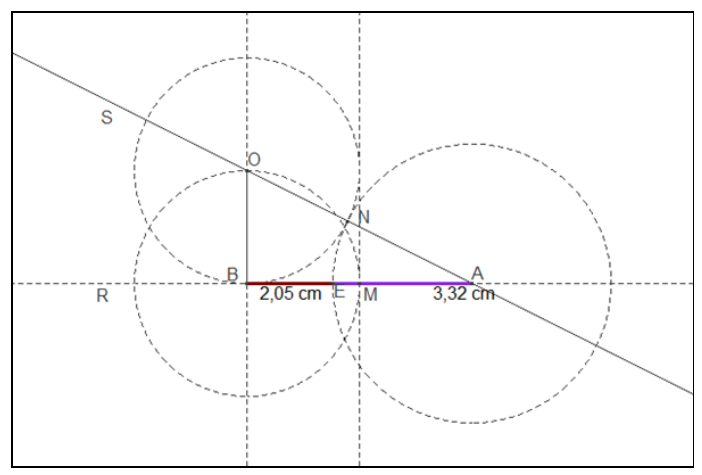

Cabe destacar que, el grupo $n^{\circ} 3$ siguió una estrategia que usualmente se emplea cuando se trabaja con una construcción geométrica con regla y compás: introducir una construcción auxiliar, la cual, consistió en ubicar un punto E sobre el segmento de extremos A y $\mathrm{B}$ tal que $\frac{A B}{A E}=\frac{A E}{E B}$; en este caso, se dice que el punto E dividió al segmento $\overline{A B}$ en razón áurea. Asimismo, este tipo de problemas permite trabajar con la construcción del rectángulo áureo, en la cual las longitudes de dos lados consecutivos del mismo están en razón áurea. Además, dado que, desde la antigua Grecia, la sección áurea ha estado vinculada con la belleza y, por ende, con el valor estético de pinturas, esculturas y construcciones; así como también con el estudio de otros temas matemáticos como los números de Fibonacci (Posamentier, 2002), se considera que la construcción seleccionada por el grupo $\mathrm{n}^{\mathrm{o}} 3$ posee un potencial didáctico que pudiera ser desarrollado en una propuesta formativa, ya que, establece vínculos con la evolución histórica de la Geometría y con otros temas matemáticos; es decir, lo planteado es una situación - problema que tiene la posibilidad de ser aprovechada por los profesores de Matemática en el diseño de tareas con contenido matemático, debido a que permite integrar distintos organizadores del currículo tales como la historia de la Matemática, la resolución de problemas y el uso de materiales y recursos. 
Cuadro 11. División de un segmento en dos segmentos cuyas longitudes están en razón áurea.

\begin{tabular}{|c|c|}
\hline Herramientas empleadas & $\begin{array}{c}\text { Comentarios } \\
\end{array}$ \\
\hline $\begin{array}{ll}\text { B } & \text { Punto } \\
\text { R } & \text { Recta: B } \\
\text { A } & \text { Punto (Punto sobre un objeto): R } \\
& \text { Segmento: B, A } \\
& \text { Recta (Mediatriz): } \\
\text { M } & \text { Punto (Punto(s) de intersección): R, _- }\end{array}$ & $\begin{array}{l}\text { Por un punto } \mathrm{B} \text {, trazan una recta } \mathrm{R} y \text {, } \\
\text { luego ubican un punto } \mathrm{A} \text {, distinto de } \mathrm{B} \text {, } \\
\text { sobre dicha recta y trazan el segmento } \mathrm{AB} \text {. } \\
\text { Seguidamente, trazan la mediatriz del } \\
\text { segmento } \mathrm{AB} \text {, siendo } \mathrm{M} \text { el punto de } \\
\text { intersección del segmento } \mathrm{AB} \text { con su } \\
\text { mediatriz. }\end{array}$ \\
\hline Recta (Recta perpendicular): B, R & $\begin{array}{l}\text { Por B trazan una perpendicular L a la } \\
\text { recta } \mathrm{R} \text {. }\end{array}$ \\
\hline $\begin{array}{ll} & \text { Segmento: M, A } \\
\text { C1 } & \text { Círculo (Compás): B, } \\
\text { O } & \text { Punto (Punto(s) de intersección): _, - } \\
& \text { Segmento: B, O } \\
\text { C2 } & \text { Círculo: O, B }\end{array}$ & $\begin{array}{l}\text { Trazan el segmento MA y la } \\
\text { circunferencia } \mathrm{C} 1 \text { con centro en } \mathrm{B} \text { y radio } \\
\mathrm{MA} \text {, donde } \mathrm{MA}=\mathrm{MB}=\mathrm{AB} / 2 \text {. } \\
\quad \mathrm{O} \text { es el punto de intersección de } \mathrm{C} 1 \text { con } \\
\text { L. Luego trazan el segmento BO y una } \\
\text { circunferencia } \mathrm{C} 2 \text { con centro en } \mathrm{O} \text { y radio } \\
\text { OB. }\end{array}$ \\
\hline $\begin{array}{ll}\mathrm{S} & \text { Recta: } \mathrm{A}, \mathrm{O} \\
\mathrm{N} & \text { Punto (Punto(s) de intersección): } \mathrm{S},{ }_{-}\end{array}$ & $\begin{array}{l}\text { Trazan la recta } \mathrm{S} \text { que pasa por A y } \mathrm{O}, \\
\text { donde } \mathrm{N} \text { es el punto de intersección de } \mathrm{C} 2 \text { y } \\
\mathrm{S} \text {. }\end{array}$ \\
\hline $\begin{array}{ll}\text { C3 } & \text { Círculo: A, N } \\
\text { E } & \text { Punto (Punto(s) de intersección): }{ }_{2},\end{array}$ & $\begin{array}{l}\text { Trazan la circunferencia C3 con centro } \\
\text { en A y radio AN. Siendo E el punto de } \\
\text { intersección de la recta R y la } \\
\text { circunferencia C3 }\end{array}$ \\
\hline 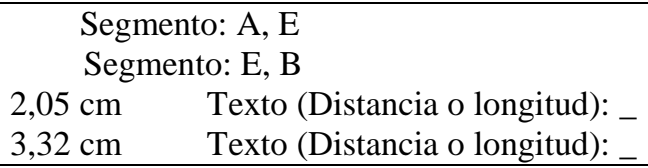 & $\begin{array}{l}\text { Trazan los segmentos AE y EB, } \\
\text { determinan sus longitudes. La razón, en la } \\
\text { que E divide al segmento AB, viene dada } \\
\text { por BE / EA o por AE / EB }\end{array}$ \\
\hline
\end{tabular}

\section{Reflexiones finales}

Una vez analizadas las producciones de los equipos en el Taller $\mathrm{n}^{\circ} 2$ sobre construcciones geométricas con doblado de papel y en un ambiente de Geometría Dinámica (AGD) y teniendo en cuenta los usos técnicos y heurísticos de un software de Geometría Dinámica según lo señalado por Iglesias \& Ortiz (2018) y Perry Carrasco, Camargo Uribe, Samper de Caicedo \& Rojas Morales (2006), así el tipo de justificaciones dadas por los participantes en el curso de Resolución de Problemas Geométricos Asistido por Computadora (RPG-AC) según lo expuesto por Balacheff (2000) (la justificación como producto final de un proceso de validación matemática puede ser vista como explicación, prueba o demostración formal) y Flores (2007) (la justificación como práctica argumentativa puede ser entendida como una actividad intelectual en la cual se ponen en juego lo que Flores denomina esquemas de argumentación), pudiera decirse que, en cuanto al uso técnico del Cabri II, los profesores en formación emplearon las herramientas correspondientes con lo descrito en el procedimiento de construcción con regla y compás, así como para mejorar la apariencia de la figura en pantalla; en cuanto al empleo de herramientas que le permitieran verificar relaciones existentes entre 
los objetos geométricos que conforman una construcción, a diferencia de lo encontrado en el Taller $\mathrm{n}^{\circ}$ 1 cuando se limitaron a medir ángulos o la longitud de un segmento (Iglesias \& Ortiz, 2019), hicieron uso del botón simetría axial, para verificar la congruencia entre figuras geométricas. Quizá esto se debió al trabajo realizado con doblado de papel, donde la congruencia entre dos figuras geométricas se estableció por superposición y su equivalente en un AGD es obtener su imagen simétrica, donde el eje de simetría se relaciona con el doblez efectuado.

En relación con el uso heurístico del Cabri II (construcción, exploración, formulación de conjeturas y validación), se encontró que los cinco grupos lograron construir la figura seleccionada, a partir de las condiciones iniciales, así como reconocer relaciones entre los objetos que la conformaban, especialmente establecieron relaciones de congruencia entre una figura y su correspondiente imagen simétrica. En los informes escritos, no formularon de forma explícita conjetura alguna; sin embargo, durante el desarrollo de las exposiciones visualizaron ciertas relaciones entre los objetos geométricos involucrados en las construcciones de un pentágono regular (grupo $\mathrm{n}^{\circ}$ 3: el punto $\mathrm{P}$ es la intersección de la mediatriz del segmento $\overline{G F}$ con la diagonal $\overline{A F}$ del rectángulo AEFD) o de un hexágono regular (grupo $\mathrm{n}^{\circ}$ 4: las medianas de los seis triángulos congruentes que conforman el hexágono regular están contenidas en las medianas del triángulo equilátero $\mathrm{ABC}$, a partir del cual iniciaron tal construcción); asimismo fueron capaces de validar las construcciones realizadas, no sólo con el Cabri II, sino también con doblado de papel.

Los grupos $\mathrm{n}^{\circ} 2$ (construcción de un triángulo equilátero) y $\mathrm{n}^{\circ} 3$ (construcción de un pentágono regular) dieron explicaciones, teniendo como referencia ciertas relaciones geométricas existentes entre los objetos que intervienen en estas construcciones y algunas definiciones y propiedades conocidas, tales como: (a) Un triángulo es equilátero si sus tres lados tienen igual longitud; (b) Una mediana de un triángulo equilátero lo descompone en dos triángulos 30 - 60 - 90 congruentes entre sí; (c) Dos figuras son congruentes, si al superponerse coinciden; (d) las mediatrices de los lados de un pentágono regular lo descomponen en dos mitades simétricas (ver figura 6). Así, por ejemplo, el grupo $\mathrm{n}^{\mathrm{o}} 2$ justifica que el triángulo BEF es equilátero, ya que, sus medianas lo descomponen en seis triángulos congruentes; para ello, efectúan varios dobleces a través de las medianas hasta que quedan superpuestos.

El grupo no 3 explicó que el pentágono A'GF'BC es regular porque al hacer coincidir dos vértices consecutivos, el doblez obtenido es la mediatriz del lado determinado por tales puntos y los lados al suponerse coinciden y, por tanto, son congruentes entre sí (ver figura 6); cuando realizaron la construcción con el Cabri II, reconocieron que GA = GF y, como los puntos $\mathrm{K}$ y $\mathrm{K}$ ' dividen en razón áurea a los segmentos $\overline{G F}$ y $\overline{G A}$ respectivamente, se llega a que $\mathrm{GK}=\mathrm{GK}$ ' (dos de los lados del pentágono); pero retoman la justificación valiéndose del doblado de papel. 
En los grupos $\mathrm{n}^{\circ} 2$ y n $^{\circ} 3$ se observa el uso de un esquema de argumentación fáctico, basado en el recuento de los procedimientos empleados, junto con un esquema de argumentación empírico, ya que, ambos grupos efectuaron la verificación empírica de relaciones de congruencia entre figuras geométricas por superposición.

El grupo $\mathrm{n}^{\circ} 1$ presentó pruebas que sirvieron para garantizar la consistencia de las correspondientes construcciones. El grupo $\mathrm{n}^{\mathrm{o}} 1$ inició la prueba, haciendo coincidir los vértices $\mathrm{B}$ y $\mathrm{D}$ del cuadrado original con el punto $\mathrm{O}$ (ver figura 2), con el propósito de superponer los triángulos EDH y EOH, así como los triángulos FBG y FOG y, así, contar con pares de lados y ángulos correspondientes congruentes. Además, en la construcción realizada con el Cabri II, la prueba se centra en el hecho que si las diagonales de un cuadrilátero se bisecan, son congruentes y perpendiculares entre sí, entonces el cuadrilátero es un cuadrado. En este grupo, se observa un esquema de argumentación analítico, apoyado en un esquema de argumentación fáctico. Esto pareciera estar relacionado con la naturaleza de la tarea propuesta que los obliga inicialmente a enfocar la atención en los procedimientos de construcción empleados, para luego tratar de reconocer relaciones entre los objetos geométricos y, por último, tratar de dar una justificación (explicación o prueba).

Cabe señalar que, aunque en esta ocasión se trabajó con el Cabri II, las actividades realizadas pueden llevarse a cabo haciendo uso de cualquier software de Geometría Dinámica (SGD) disponible, haciendo los ajustes que sean necesarios; actualmente, en el curso de RPG-AC, se está utilizando el GeoGebra, ya que, es un software libre, con versiones disponibles para distintas plataformas y dispositivos tecnológicos, que favorece el manejo de distintos sistemas de representación. Además, se considera que el combinar el uso tanto del doblado de papel como de un SGD favorece el reconocimiento de las relaciones existentes entre los objetos que intervienen en una construcción, así como la validación del procedimiento de construcción empleado. Por otra parte, el contar con unos axiomas para el doblado de papel (reglas en las cuales se soportan las operaciones que son posibles realizar en una construcción con doblado de papel) y, además, reconocer y describir su fundamentación y relación con definiciones, axiomas y teoremas de la Geometría Euclidiana es lo que nos ha permitido examinar la equivalencia entre dos maneras de construir una figura geométrica; es decir, se ha analizado y se ha logrado mostrar a lo largo de este trabajo, las actividades y experiencias formativas llevadas a cabo por el grupo que participó en el estudio, las cuales contribuyen a la formación didáctica y matemática de los futuros profesores.

Finalmente, se considera que, en el contexto de la formación inicial de profesores de Matemática, es necesario que los estudiantes realicen tareas, como las aquí descritas, en las cuales tengan que poner en juego sus conocimientos didáctico - matemáticos, los cuales más adelante les 
permitirán diseñar y gestionar tareas matemáticas en el ámbito escolar en donde les corresponda laborar.

\section{Referencias}

Alsina Catalá, C., Fortuny Aymemí, J.M. \& Pérez Gómez, R. (1997). ¿Por qué Geometría? Propuestas didácticas para la ESO. Madrid: Síntesis.

Arrieche, B. \& Iglesias, M. (2010). Explorando ángulos e triángulos com dobraduras em papel. Boletim GEPEM, 57, 105-117.

Avilés Fajardo, P. I. (2016). Uso de la didáctica del plegado de papel, como herramienta de apoyo en la enseñanza de los contenidos de la Geometría para estudiantes del $10^{\circ}$ año de Educación General Básica, de la Unidad Educativa Best del Cantón Vinces. Tesis previa a la obtención del Título de Magister en Ciencias de la Educación. Quito: Pontificia Universidad Católica del Ecuador. Recuperado el 13 de enero de 2019, de http://repositorio.puce.edu.ec/bitstream/handle/22000/12227/TESIS.pdf?sequence=1

Balacheff, N. (2000). Procesos de Prueba en los alumnos de Matemática. Bogotá: Una Empresa Docente de la Universidad de los Andes.

Chocarro Pernaut, A. (2016). Números de Papel. Trabajo de fin de grado. Logroño: Facultad de Ciencia y Tecnología de la Universidad de La Rioja. Recuperado el 13 de enero de 2019, de https://biblioteca.unirioja.es/tfe_e/TFE002205.pdf

Flores, A.H. (2007). Esquemas de Argumentación en Profesores de Matemáticas del Bachillerato. Educación Matemática, 19 (1), 63-98.

Gutiérrez, A. \& Jaime, A. (2012). Reflexiones sobre la enseñanza de la geometría en primaria y secundaria. Tecné, Episteme y Didaxis, 32, 55 - 70.

Iglesias, M. \& Ortiz, J. (2018). Usos del software de geometría dinámica en la formación inicial de profesores de matemáticas. Matemáticas, Educación y Sociedad, 1(2), 21-35.

Iglesias, M. \& Ortiz, J. (2019). La demostración en Geometría desde una perspectiva didáctica. Unión 55, abril 2019, 159-183.

Perry Carrasco, P., Camargo Uribe, L., Samper de Caicedo, C. \& Rojas Morales, C. (2006). Actividad demostrativa en la formación inicial del profesor de matemáticas. Bogotá: Fondo Editorial de la Universidad Pedagógica Nacional.

Posamentier, A. S. (2002). Advanced Euclidean Geometry. USA: Key College Publishing.

Royo Prieto, J.I. (2002). Matemáticas y Papiroflexia. Sigma n ${ }^{\circ} 21,175$ - 192. Recuperado el 13 de enero de 2019, de http://www.hezkuntza.ejgv.euskadi.eus/r43573/es/contenidos/informacion/dia6_sigma/es_sigma/adjuntos/sigma_21/11_matematic as_y_papiroflexia.pdf

Sánchez, R. (2008). El plegado de papel y las construcciones con regla y compás en la enseñanza y el aprendizaje de la geometría del triángulo a nivel de $7^{\circ}$ grado de educación básica. Trabajo de grado de maestría no publicado. Universidad Pedagógica Experimental Libertador, Instituto Pedagógico Rafael Alberto Escobar Lara, Maracay. 
Van Hiele, P.M. (1957). El problema de la comprensión. En conexión con la comprensión de los escolares en el aprendizaje de la geometría. Tesis doctoral no publicada. Universidad Real de Utrecht: Utrecht, Holanda. Recuperado el 11 de octubre de 2018, de http://www.uv.es/Angel.Gutierrez/aprengeom/archivos2/VanHiele57.pdf

Van Hiele, P.M. (1959). La pensée de l'enfant et la géométrie. Bulletin de l'APMEP 198, 199205. Traducido al español por Ricardo Barroso. Recuperado el 11 de octubre de 2018, de http://www.uv.es/Angel.Gutierrez/aprengeom/aprgeorefer.html 